\title{
New active galactic nuclei detected in ROSAT All Sky Survey galaxies
}

\section{The complete dataset ${ }^{\star, \star \star, \star \star \star}$}

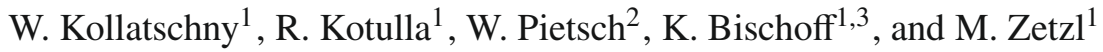 \\ ${ }^{1}$ Institut für Astrophysik, Universität Göttingen, Friedrich-Hund Platz 1, 37077 Göttingen, Germany \\ e-mail: wkollat@astro.physik.uni-goettingen.de \\ 2 Max-Planck-Institut für Extraterrestrische Physik, Giessenbachstrasse, 85740 Garching, Germany \\ 3 Halfmann Teleskoptechnik GmbH \& Co. KG, Gessertshausener Str. 8, 86356 Neusäß-Vogelsang, Germany
}

Received 27 August 2007 / Accepted 15 March 2008

\section{ABSTRACT}

\begin{abstract}
Aims. The ROSAT ALL Sky Survey Bright Source Catalogue (RASS-BSC) has been correlated with the Catalogue of Principal Galaxies (PGC) to identify new extragalactic counterparts. 550 reliable optical counterparts have been detected. However there existed no optical spectra for about 200 Active Galactic Nuclei (AGN) candidates before the ROSAT ALL Sky Survey (RASS) was completed.

Methods. We took optical spectra of 176 X-ray candidates and companions at ESO, Calar Alto observatory and McDonald observatory. When necessary we used a line profile decomposition to measure line fluxes, widths and centers to classify their type of activity. Results. We discuss the redshift-, linewidth-, as well as optical and X-ray luminosity distribution of our ROSAT selected sample. 139 galaxies of our 166 X-ray counterparts have been identified as AGN with 93 being Seyfert 1 galaxies (61\%). Eighteen of them $(20 \%)$ are Narrow Line Seyfert 1 galaxies. 34 X-ray candidates (21\%) are LINERs and only eight candidates (5\%) are Seyfert 2. The ratio of the number of Seyfert 1 galaxies to Seyfert 2 galaxies is about 11/1. Optical surveys result in ratios of 1/1.4. The high fraction of detected Seyfert 1 galaxies is explained by the sensitivity of the ROSAT to soft X-rays which are heavily absorbed in type 2 AGN. Two X-ray candidates are HII-galaxies and 25 candidates (15\%) show no signs of spectral activity. The AGN in our RASS selected sample exhibit slightly higher optical luminosities $\left(M_{B}=(-20.71 \pm 1.75)\right.$ mag $)$ and similar X-ray luminosities $\left(\log \left(L_{\mathrm{X}}\left[\mathrm{erg} \mathrm{s}^{-1}\right]\right)=42.9 \pm 1.7\right)$ compared to other AGN surveys. The $\mathrm{H} \alpha$ line width distribution $(F W H M)$ of our newly identified ROSAT AGN sample is similar to the line widths distribution based on SDSS AGN. However, our newly identified RASS AGN have rather reddish colors explaining why they have not been detected before in ultraviolet or blue excess surveys.
\end{abstract}

Key words. X-rays: galaxies - galaxies: active - surveys

\section{Introduction}

Canonical active galactic nuclei (AGN) are blue, UV-excess sources. They are known to be strong X-ray emitters (e.g. Fabbiano et al. 1992) in comparison to normal galaxies. However, not all AGN detected by their optical, infrared or radio properties have counterparts in X-ray surveys. The X-ray satellite ROSAT detected nearly 19000 bright sources in the soft X-ray band (0.1-2.4 keV). These are listed in the ROSAT All Sky Survey Bright Source Catalogue (RASS-BSC) (Voges et al. 1993, 1999). We are interested in the optical spectral properties of ROSAT-selected AGN. On the one hand we wanted to take optical spectra of as many ROSAT counterparts as possible. On the other hand we wanted to test whether our newly identified RASS selected AGN show spectral properties different to those AGN that have been detected earlier with other

* Based on observations collected at European Southern Observatory, Chile.

$\star \star$ Based on observations taken at the German-Spanish Astronomical Center, Calar Alto, operated by the Max-Planck-Institute for Astronomy, Heidelberg jointly with the Spanish Commission for Astronomy.

$\star \star \star$ Based on observations collected at McDonald Observatory. strategies. A correlation of the RASS with optical counterparts in the Principal Catalog of Galaxies (Paturel et al. 1989, 2003) resulted in about 900 extragalactic counterparts or candidates. These X-ray sources are mainly AGN or nearby galaxies. However, for about 200 AGN candidates no optical spectra existed when the ROSAT ALL Sky Survey (RASS) was completed.

This paper is the second in a series describing the optical spectra and the physical properties of these AGN. In the first paper the selection strategy was described by Pietsch et al. (1998, hereafter Paper I) and the optical spectra of 35 X-ray counterparts were presented.

In parallel to our new optical identifications we investigated $\mathrm{X}$-ray and ROSAT-related AGN papers to determine whether some of our targets had been identified since (Moran et al. 1996; Simcoe et al. 1997; Bade et al. 1998; Motch et al. 1998; Appenzeller et al. 1998; Fischer et al. 1998; Bauer et al. 2000; Brinkmann et al. 2000; Vaughan et al. 2001; Xu et al. 2001; Grupe et al. 2004). Furthermore we checked SDSS-related papers (Adelman et al. 2006) as well as the Veron-Cetty catalogue of AGN (2006). In our list of 166 new AGN candidates we found some information with respect to 41 objects. In a preprint (Bischoff et al. 1999) we presented some preliminary data of 17 of our new AGN candidates. 


\section{Observations}

\subsection{The ROSAT AGN sample}

In Paper I we described in detail our strategy to find new AGN in the ROSAT All Sky Survey. Further detailed information about the galaxy identification project can be found in Zimmermann et al. (2001). The selection is based on the empirical finding that most active galaxies are strong X-ray emitters in comparison to non-active galaxies. In the RASS-BSC (Voges 1999) nearly 19000 X-ray sources are listed, having count rates in excess of $0.05 \mathrm{cts} / \mathrm{s}$ : these objects were subsequently cross-correlated with the Principal Catalog of Galaxies (Paturel et al. 1989, 2003). 904 X-ray sources were identified as having extragalactic counterparts.

Extended X-ray sources such as early-type galaxies or clusters of galaxies were rejected from our sample in the next step.

Finally, 547 correlations have been determined as reliable counterparts of compact extragalactic X-ray sources. These sources were cross-checked with the NASA Extragalactic Database (NED): 349 sources were known active galaxies. However, no spectral information existed for 198 AGN candidates. Our goal was to obtain spectra of as many of these objects as possible.

\subsection{Optical observations}

Table 2 contains information on our observed sample of $166 \mathrm{X}$-ray counterparts as well as some information on ten additional companions of theses counterparts. Given are the ROSAT name (1), the optical positions derived from the Digitized Sky Survey (DSS) (2 and 3), the object name (4), the X-ray count rate (5), derived X-ray luminosity (6), the apparent (7) and absolute (8) $B$-band magnitudes (for details see Paper I).

Figure 1 shows the spatial distribution - in equatorial coordinates - of all AGN candidates for which we have acquired spectra. They are distributed homogeneously over the whole sky, except for the regions blocked by the Milky Way. The sky distribution of all 904 extragalactic X-ray counterparts has been shown by Zimmermann et al. (2001, their Fig. 3).

We obtained optical spectra of our X-ray candidate galaxies during five observing campaigns. We used the $2.2 \mathrm{~m}$ telescope at Calar Alto Observatory in Spain, the $2.2 \mathrm{~m}$ telescope at La Silla/ESO in Chile, as well as the $2.7 \mathrm{~m}$ telescope at McDonald Observatory in Texas. We obtained spectra of 176 AGN candidates and companion galaxies. In a few cases we observed more than one object when there was more than one candidate in the ROSAT error box. We identified 166 AGN and non-AGN as optical counterparts of ROSAT RASS sources. Details of the observations, such as the observing periods, the telescopes and spectrographs we used, as well as the wavelength coverage of the spectra are listed in Table 1.

The observing dates of the individual galaxies and exposure times are listed in Table 3. Exposure times range from 10 to $45 \mathrm{~min}$. The spectrograph slits had projected widths of 1.5 to 2 arcsec. We had typical seeing conditions of 1 to 1.5 arcsec. The slit was oriented in a north-south direction, in most cases, to minimize the impact of light losses caused by differential refraction.

Our spectra typically cover a wavelength range from $3800 / 4700 \AA$ to $7300 / 8200 \AA$ (see Table 1) with a spectral resolution of 8 to $10 \AA$. Additional spectra were taken after each object exposure for wavelength calibration. Various standard stars were observed for flux calibration.

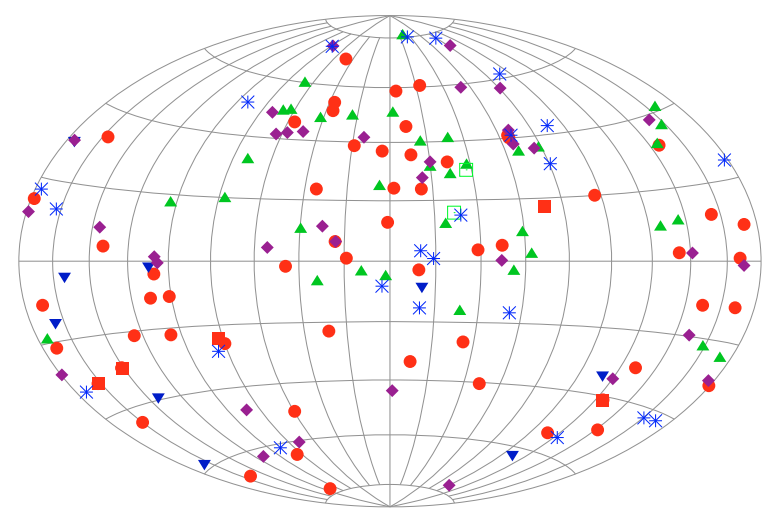

Fig. 1. Aitoff projection of all selected sources in J2000 equatorial coordinates (Center of the plot: RA $=12 \mathrm{~h}, \mathrm{Dec}=0^{\circ}$; RA increases to the right.). There is a homogenous spatial distribution of our X-ray selected galaxies, except for the regions blocked by the Milky Way. The symbols used in this and all following plots: Sy 1 type objects: filled red circles, intermediate types (Sy 1.5/1.8/1.9): green upward triangles, Sy 2: blue downward triangles, LINERS: purple diamonds, H II: green open squares, non-active: blue stars.

Table 1. List of our observing runs giving the telescopes and instruments used for the acquisition of the spectra as well as their wavelength range.

\begin{tabular}{|c|c|c|c|c|}
\hline \# & Observing run & Telescope & Instrument (CCD) & $\lambda \lambda[\AA]$ \\
\hline 1 & $96 / 11 / 02-06$ & ESO $2.2 \mathrm{~m}^{a}$ & EFOSC2 (LORAL) & $3800-8000$ \\
\hline 2 & 97/07/02-06 & ESO $2.2 \mathrm{~m}^{a}$ & EFOSC2 (LORAL) & $4100-7400$ \\
\hline 3 & $97 / 07 / 28-08 / 01$ & CA $2.2 \mathrm{~m}^{b}$ & CAFOS (SITe\#1d) & $4200-8200$ \\
\hline 4 & $98 / 03 / 01-05$ & CA $2.2 \mathrm{~m}^{b}$ & CAFOS (SITe\#1d) & $4200-8200$ \\
\hline 5 & 00/02/08-09 & MDO $2.7 \mathrm{~m}^{c}$ & LCS (TK3/CC1) & $4700-7300$ \\
\hline
\end{tabular}

${ }^{a}$ La Silla, Chile.

${ }^{b}$ Calar Alto, Spain.

${ }^{c}$ McDonald Observatory, Texas, USA.

\subsection{Data reduction}

The reduction of the spectra (bias subtraction, cosmic ray correction, flat-field correction, 2D-wavelength calibration, night sky subtraction, flux calibration) was done in a homogeneous way with the IRAF reduction packages ${ }^{1}$. We extracted spectra of the central 3 arcsec. The wavelength calibration was done using comparison spectra of $\mathrm{He}-\mathrm{Ar}$ (runs \#1, \#2 ), $\mathrm{Hg}-\mathrm{Cd}-\mathrm{He}$ (\#3, $\# 4$ ) and $\mathrm{He}-\mathrm{Ne}(\# 5)$. The flux calibration was done by means of the standard stars HD 49798 (\#1), NGC 7293 (\#1, Turnshek et al. 1990), BD 332642 (\#4) and G191B2B (\#4,5).

Redshifts, emission line intensities and line widths were derived from individual emission/absorption lines as well as determined by fitting line complexes ( $\mathrm{H} \alpha$ narrow and broad, [N II], [S II]; H $\beta$ narrow and broad). Only the narrow Balmer line components were used for the diagnostic diagram (see Fig. 7) to derive their activity type.

\footnotetext{
1 IRAF is distributed by the National Optical Astronomy Observatories, which are operated by the Association of Universities for Research in Astronomy, Inc., under cooperative agreement with the National Science Foundation.
} 
Table 2. X-ray identification information.

\begin{tabular}{|c|c|c|c|c|c|c|c|}
\hline ROSAT-name & RA, Dec & $2000.0)$ & Name & Countrate & $\log \left(L_{X}\right)$ & & $M_{B}$ \\
\hline & {$[\mathrm{h} \mathrm{m} \mathrm{s}]$} & {$[\mathrm{d} \mathrm{m} \mathrm{s}]$} & & & {$\left[\mathrm{ergs}^{-1}\right]$} & & \\
\hline (1) & (2) & (3) & (4) & (5) & (6) & (7) & (8) \\
\hline 1RXS J000156.7 - 273748 & 000155.8 & -273738 & ESO 409-003 & $0.067 \pm 0.0171$ & 42.55 & 14.66 & -20.63 \\
\hline 1RXS J000459.1 + 114205 & 000458.4 & 114203 & UGC 32 & $0.148 \pm 0.0205$ & 43.77 & 14.20 & -23.20 \\
\hline 1RXS J000805.6 + 145027 & 000805.6 & 145023 & CGCG 433-025 & $0.179 \pm 0.0180$ & 43.64 & 15.70 & -20.60 \\
\hline 1RXS J001530.2 + 172009 & 001530.9 & 171942 & NGC 57 & $0.072 \pm 0.0198$ & 42.23 & 13.14 & -21.23 \\
\hline 1RXS J001823.8 + 300357 & 001723.6 & 300848 & NGC 71 & $0.006 \pm 0.0202$ & 42.40 & 14.42 & -20.36 \\
\hline 1RXS J001823.8 + 300357 & 001822.8 & 300447 & NGC 70 & $0.006 \pm 0.0202$ & 42.30 & 14.18 & -20.71 \\
\hline 1RXS J002108.1 - 190950 & 002113.2 & -191044 & PKS 0018-19 & $0.104 \pm 0.0195$ & 43.81 & 17.00 & -20.95 \\
\hline 1RXS J002534.9 - 330255 & 002531.3 & -330248 & ESO 350-015 & $0.249 \pm 0.0310$ & 43.62 & 14.23 & -22.31 \\
\hline 1RXS J003413.7 - 212619 & 003413.5 & -212620 & HCG 4a & $0.330 \pm 0.0350$ & 43.14 & 13.70 & -21.43 \\
\hline 1RXS J004236.9 - 104919 & 0042367 & -104922 & VIII Zw 36 & $0.244 \pm 0.0280$ & 43.55 & 14.60 & -21.51 \\
\hline 1RXS J010013.4 - 151755 & 010015.9 & -151757 & MCG -03-03-017 & $.151 \pm 0.0207$ & 43.47 & 14.90 & -21.80 \\
\hline 1RXS J010517.5 - 582618 & 010516.5 & -582613 & ESO 113-010 & $.182 \pm 0.0390$ & 43.40 & 14.60 & -20.47 \\
\hline 1RXS J010918.0 + 1 & 010918.4 & 131008 & UGC 716 & $.069 \pm$ & 43.45 & 15.60 & -21.30 \\
\hline 1RXS J011219.5 - 3 & 011219.2 & -320344 & NGC 427 & $.153 \pm$ & 42.88 & 15.10 & -20.55 \\
\hline $11233.3-3$ & 11232.6 & -320144 & RX J011232.8-320 & $0.033 \pm$ & & 21.10 & \\
\hline 1RXS J0 & 12019.6 & -440743 & ESO 244-017 & $305 \pm$ & 43.12 & 14.60 & -20.28 \\
\hline $13124.2+3$ & 13123.8 & 330838 & KUG 012 & $.104 \pm$ & 43.56 & 16.50 & -20.78 \\
\hline 1RXS J014526.6 - 0 & 014525.3 & -034939 & MCG -01- & $175 \pm$ & 42.70 & 13.30 & -21.00 \\
\hline 1RXS J014739.5 - 660952 & 014739.5 & -660949 & ESO 080-005 & $0.091 \pm 0.0200$ & 42.32 & 16.00 & -19.17 \\
\hline 1RXS J023513.9 - 293616 & 023513.4 & -293618 & ESO 416-002 & $0.356 \pm 0.0340$ & 43.56 & 14.90 & -22.00 \\
\hline 1RXS J023536.7 - 293845 & 023536.6 & -293844 & PHL 1389 & $0.043 \pm 0.0040$ & & 16.10 & \\
\hline 1RXS J025552.4 + 091853 & 025552.2 & 091842 & IC 1867 & $0.083 \pm 0.0190$ & 43.02 & 14.60 & -20.46 \\
\hline 1RXS J030606.3 - 3 & 030605.9 & -300210 & NGC 1217 & $0.193 \pm 0.0230$ & 42.77 & 13.30 & -21.28 \\
\hline 1RXS J030825.9 + 040637 & 030826.3 & 040640 & NGC 1218 & $0.175 \pm 0.0210$ & 43.35 & 13.90 & -21.41 \\
\hline 1RXS J034203.8 - 211428 & 034202.8 & -211426 & & $0.258 \pm 0.0260$ & 42.63 & 12.80 & -20.90 \\
\hline 1RXS J042710.2-62 & 042712.5 & -624709 & & 50 & 42.41 & 13.00 & -21.33 \\
\hline 1RXS J043520.2 - 7 & 043516.2 & -780157 & & & 44.10 & 14.20 & -22.76 \\
\hline $1 \mathrm{RXS}$ & & -104745 & $\mathrm{M}$ & & 43.08 & 15.20 & -20.60 \\
\hline $1 \mathrm{R}\rangle$ & 4148.3 & -011810 & & & 42.93 & 14.20 & -21.13 \\
\hline $1 \mathrm{R}\rangle$ & 5141.4 & -034834 & MC & 290 & 42.90 & 14.60 & -19.42 \\
\hline 1RXS J & 045258.9 & 011953 & UGC 31 & 140 & 42.03 & 14.90 & -20.35 \\
\hline $45840.4-2$ & 045840.3 & -215932 & ESO 552 & $0.106 \pm$ & 43.47 & 16.30 & -19.72 \\
\hline $45859.6-002904$ & 045854.6 & -002920 & NGC 171 & $0.120 \pm$ & 41.84 & 13.30 & -20.59 \\
\hline $50820.9+172134$ & 50820.5 & 172158 & CGCG 46 & $0.060 \pm 0.0128$ & 41.77 & 15.40 & -19.05 \\
\hline $1 \mathrm{RXS} \mathrm{J}$ & 051621.2 & -103340 & MCG -0 & 290 & 43.64 & 15.50 & -19.79 \\
\hline $15.3+5$ & 054714.9 & 505215 & & 144 & 42.01 & 15.60 & -19.50 \\
\hline 1RXS J055559.4 - 612438 & 055552.6 & -612414 & ESO 120 & $.093 \pm 0.0070$ & 43.23 & 15.30 & -20.60 \\
\hline 1RXS J060635.4 - 472957 & 060635.8 & -472956 & & $0.213 \pm 0.0160$ & 43.25 & 14.60 & -20.79 \\
\hline 1RXS J062122.8 - 280712 & 062126.2 & -280653 & & $0.077 \pm 0$ & 41.95 & 13.40 & -21.38 \\
\hline 1RXS J062307.7 - 643618 & 062307.7 & -643621 & & & 44.84 & 14.80 & -23.82 \\
\hline 1RXS J0 & 59.4 & -24 & & & & 15.40 & \\
\hline & 4011.8 & -25 & & & 43.24 & 14.10 & -20.90 \\
\hline $1 \mathrm{R}\rangle$ & 4714.0 & 741412 & $\mathrm{~N}$ & & 41.76 & 14.00 & -20.19 \\
\hline $1 \mathrm{R}\rangle$ & 064745.8 & 742854 & NGC 2258 & & 41.45 & 13.00 & -20.67 \\
\hline $1 \mathrm{R}\rangle$ & 065215.1 & 193157 & $\mathrm{C}$ & 53 & 42.90 & 15.60 & -21.36 \\
\hline 1RXS J070907.6 + 4 & 070908.0 & 493656 & NGC 232 & $.106 \pm 0$. & 42.01 & 13.10 & -21.37 \\
\hline 1RXS J071148.0 + 321902 & 071147.7 & 321836 & B2 $0708+32 b$ & $.332 \pm 0.0293$ & 43.58 & 15.80 & -21.35 \\
\hline 1RXS J071204.2 - 603005 & 071203.3 & -603030 & ESO 122-016 (N & $.106 \pm 0.1000$ & 43.30 & 14.60 & -21.00 \\
\hline 1RXS J073353.4 + 4C & 073353.1 & 491731 & UGC 39 & $.080 \pm 0.0164$ & 41.98 & 15.30 & -19.40 \\
\hline $73727.1+594143$ & 073730.1 & 594103 & UGC 392 & & 42.40 & 15.50 & -20.61 \\
\hline 1RXS J074701.7 + 413211 & 074702.0 & 413210 & UGC 4018 & $0.077 \pm 0.0143$ & 42.19 & 14.80 & -20.52 \\
\hline 1RXS J075151.6 + 494854 & 075151.9 & 494852 & MCG +08-15-09 & $0.067 \pm 0.0141$ & 42.01 & 15.10 & -19.93 \\
\hline 1RXS J080157.7 - 494639 & 080157.9 & -494642 & ESO 209-012 & $0.163 \pm 0.0160$ & 44.19 & 15.30 & -20.77 \\
\hline 1RXS J081021.3 + 421657 & 081023.3 & 421626 & CGCG 207-040 & $0.138 \pm 0.0215$ & 43.14 & 15.60 & -21.48 \\
\hline 1RXS J081517.8 + 460429 & 081516.9 & 460431 & MGC +08-15-56 & $0.138 \pm 0.0190$ & 42.76 & 15.20 & -20.95 \\
\hline 1RXS J082321.4 + 042231 & & 042221 & & & 42.30 & 14.80 & -20.80 \\
\hline 1RXS J083539.1 - 040508 & 083538.8 & -040517 & NGC 2617 & & 41.99 & 14.00 & -19.93 \\
\hline 1RXS J084456.2 + 425826 & 084456.6 & 425835 & MCG +07-18- & & 42.56 & 15.00 & -21.70 \\
\hline 1RXS J085001.4 + 701804 & 085002.3 & 701806 & QSO east of NGC 2650 & $0.057 \pm 0.0144$ & 46.01 & 16.00 & -28.91 \\
\hline 1RXS J085617.7 - 013809 & 085617.8 & -013908 & CGCG 005-037 & $0.128 \pm 0.0219$ & 42.95 & 15.50 & -21.41 \\
\hline $91345.4+474208$ & 091345.4 & 474206 & MCG +08-17-60 & $0148+0$ & 42.82 & 15.90 & -20.77 \\
\hline & 092115.5 & 101741 & VIII Zw 45 & $0.141 \pm 0.0296$ & 42.69 & 15.40 & -20.65 \\
\hline $93308.2+5$ & 093308.9 & 534749 & KUG 0929+540 & $0.067 \pm 0.0181$ & 42.56 & 17.00 & -19.85 \\
\hline 1RXS J093642.6 + 505249 & 093643.1 & 505249 & KUG 0933+511 & $0.054 \pm 0.0133$ & 42.40 & 15.50 & -21.29 \\
\hline
\end{tabular}


Table 2. continued.

\begin{tabular}{|c|c|c|c|c|c|c|c|}
\hline \multirow{3}{*}{$\begin{array}{l}\text { ROSAT-name } \\
\text { (1) }\end{array}$} & \multicolumn{2}{|c|}{ RA, Dec (J2000.0) } & \multirow{3}{*}{$\begin{array}{l}\text { Name } \\
\text { (4) }\end{array}$} & \multirow{3}{*}{$\begin{array}{c}\text { Countrate } \\
{\left[\text { cts s}^{-1}\right]} \\
(5)\end{array}$} & \multirow{3}{*}{$\begin{array}{r}\log \left(L_{X}\right) \\
{\left[\mathrm{ergs}^{-1}\right]} \\
(6)\end{array}$} & \multirow{3}{*}{$\begin{array}{r}m_{B} \\
{[\mathrm{mag}]} \\
(7)\end{array}$} & \multirow{3}{*}{$\begin{array}{r}M_{B} \\
{[\mathrm{mag}]} \\
(8)\end{array}$} \\
\hline & & {$[\mathrm{d} \mathrm{m} \mathrm{s}]$} & & & & & \\
\hline & (2) & (3) & & & & & \\
\hline 1RXS J094204.0 + 234106 & 094204.8 & 234107 & CGCG 122-055 & $0.120 \pm 0.0181$ & 42.06 & 15.30 & -19.49 \\
\hline 1RXS J095154.9 - 064916 & 095155.0 & -064923 & NGC 3035 & $0.263 \pm 0.0274$ & 42.14 & 13.50 & -20.42 \\
\hline 1RXS J095948.0 + 112926 & 095946.8 & 112820 & CGCG 064-024 & $0.159 \pm 0.0238$ & 43.31 & 15.70 & -21.80 \\
\hline 1RXS J100554.9 - 230318 & 100555.4 & -230325 & ESO 499-041 & $0.131 \pm 0.0188$ & 41.71 & 13.40 & -20.15 \\
\hline 1RXS J102403.1 + 062954 & 102359.8 & 062908 & CGCG 037-022 & $0.119 \pm 0.0198$ & 42.66 & 16.00 & -20.28 \\
\hline 1RXS J102445.5 + 062455 & 102443.5 & 062603 & CGCG 037-028 & $0.052 \pm 0.0141$ & 42.27 & 15.50 & -20.75 \\
\hline 1RXS J102841.7 + 490425 & 102842.0 & 490419 & CGCG 240-047 & $0.073 \pm 0.0138$ & 42.33 & 15.30 & -20.95 \\
\hline 1RXS J104333.4 + 010109 & 104332.9 & 010109 & GNB 069 & $0.070 \pm 0.0183$ & 42.91 & 16.80 & -20.54 \\
\hline 1RXS J104439.4 + 384541 & 104439.2 & 384534 & CGCG 212-045 & $0.351 \pm 0.0303$ & 42.89 & 15.00 & -20.92 \\
\hline 1RXS J110312.1 + 414200 & 110311.0 & 414219 & MCG +07-23-15 & $0.117 \pm 0.0216$ & 42.22 & 15.10 & -20.41 \\
\hline 1RXS J110941.7 - 033915 & 110942.9 & -034903 & CGCG 011-012 & $0.161 \pm 0.0243$ & 42.76 & 15.40 & -20.59 \\
\hline 1RXS J114014.0 + 244150 & 114013.9 & 244149 & NGC 3798 & $0.065 \pm 0.0137$ & 41.20 & 13.90 & -19.51 \\
\hline 1RXS J114429.9 + 365314 & 114429.9 & 365308 & KUG $1141+371$ & $1.368 \pm 0.0740$ & 43.53 & 16.50 & -19.48 \\
\hline 1RXS J114604.3 - 081550 & 114903.8 & -081604 & IC 734 & $0.080 \pm 0.0183$ & 43.07 & 16.00 & -21.55 \\
\hline 1RXS J115238.2 - 051229 & 115236.2 & -051225 & MCG -01-30-041 & $0.108 \pm 0.0190$ & 42.41 & 13.80 & -20.62 \\
\hline 1RXS J115537.0 + 125251 & 115535.2 & 125219 & VIII Zw & $0.134 \pm 0.0291$ & 43.54 & 17.90 & -20.58 \\
\hline 1RXS J120452.8 - 434353 & 120452.9 & -434354 & ESO 267-013 & $0.110 \pm 0.0279$ & 42.19 & 14.15 & -19.68 \\
\hline 1RXS J120655.0 + 501744 & 120655.6 & 501737 & MCG +08-22-71 & $0.074 \pm 0.0160$ & 42.70 & 17.00 & -20.03 \\
\hline 1RXS J120719.4 + 241204 & 120719.8 & 241156 & Mrk 648 & $0.196 \pm 0.0235$ & 42.93 & 16.00 & -20.61 \\
\hline 1RXS J121754.7 + 583936 & 121755.0 & 583935 & Mrk 202 & $0.213 \pm 0.0246$ & 42.25 & 15.60 & -19.33 \\
\hline 1RXS J123651.1 + 453907 & 123651.2 & 453904 & MCG +08-23-67 & $0.526 \pm 0.0356$ & 42.89 & 15.20 & -20.31 \\
\hline 1RXS J124046.8 - 333408 & 124047.0 & -333412 & ESO 381-007 & $0.446 \pm 0.0455$ & 43.95 & 15.94 & -20.80 \\
\hline 1RXS J124306.5 + 353859 & 124307.4 & 353905 & KUG $1240+359$ & $0.088 \pm 0.0158$ & 44.68 & 16.50 & -25.43 \\
\hline 1RXS J125042.5 - 024929 & 125042.4 & -024931 & CGCG 015-026 & $0.068 \pm 0.0164$ & 43.01 & 15.70 & -20.71 \\
\hline 1RXS J125253.5 - 152445 & 125252.6 & -152448 & NGC 4756 & $0.068 \pm 0.0185$ & 41.43 & 13.00 & -20.63 \\
\hline 1RXS J125347.3 + 032620 & 125347.0 & 032630 & CGCG 043-056 & $0.186 \pm 0.0422$ & 43.73 & 15.70 & -21.42 \\
\hline 1RXS J125609.5 - 080906 & 125610.1 & -080905 & MCG -01-33-054 & $0.164 \pm 0.0259$ & 42.27 & 14.54 & -19.07 \\
\hline 1RXS J125851.4 + 235532 & 125851.5 & 235526 & KUG 1256+241 & $0.430 \pm 0$ & 43.56 & 17.00 & -20.43 \\
\hline 1 RXS J130158.9+274708 & 130200.1 & 274658 & CGCG 160-104 & $0.124 \pm 0$ & 42.00 & 15.40 & -19.61 \\
\hline 1RXS J130456.7 + 3 & 130457.0 & 395530 & 65 & 19 & 41.79 & 15.00 & -20.27 \\
\hline $\mathrm{J} 131633.1+$ & 131627.0 & 005112 & VIII Zw 2 & $0.053 \pm 0$. & 42.75 & 17.30 & -20.18 \\
\hline 1RXS J131905.8 + 310854 & 131906.0 & 310853 & CGCG 1 & $0.113 \pm 0$ & 42.23 & 15.60 & -20.01 \\
\hline 1RXS J132016.3 + 330828 & 132014.6 & 330840 & NGC 5098 & $0.180 \pm 0.0214$ & 42.51 & 15.00 & -20.85 \\
\hline 1RXS J133151.1 + 603451 & 133150.7 & 603448 & MCG +10-19-84 & $0.125 \pm 0.0156$ & 43.45 & 16.00 & -22.43 \\
\hline 1RXS J133908.5 + 115855 & 133908.5 & 115854 & VIII Zw 327 & $0.133 \pm 0.0227$ & 43.30 & 17.80 & -20.10 \\
\hline 1RXS J134352.7 + 803544 & 134354.5 & 803558 & CGCG 365-016 & $0.050 \pm 0.0098$ & 42.35 & 15.40 & -20.90 \\
\hline 1RXS J135420.2 + 325547 & 135320.0 & 325548 & UGC 882 & $835 \pm 0.0459$ & 42.93 & 14.80 & -20.36 \\
\hline 1RXS J135536.3 + 155755 & 135537.0 & 155814 & CGCG 103-009 & $0.112 \pm 0.0206$ & 41.69 & 15.70 & -18.43 \\
\hline 1RXS J135625.3 + 283138 & 135625.3 & 283135 & $\mathrm{MCG}+05-33-24 \mathrm{a}$ & $0.059 \pm 0.0147$ & 42.69 & 15.60 & -21.84 \\
\hline 1RXS J140543.6 + 405115 & 140544.4 & 405116 & CGCG 219-050 & $0.164 \pm 0.0207$ & 43.03 & 15.70 & -21.54 \\
\hline 1RXS J140619.7 - 163721 & 140620.7 & -163700 & NPM1G - 16.0443 & $0.057 \pm 0.0155$ & 42.50 & 16.09 & -19.25 \\
\hline 1RXS J140723.3 + 150447 & 140722.3 & 150439 & CGCG 103-073 & $0.139 \pm 0.0$ & 42.14 & 14.60 & -20.49 \\
\hline 1 RXS J141802.6 + 800710 & 141749.0 & 800654 & CGCG 3 & 07 & 42.39 & 15.30 & -21.04 \\
\hline $.5-263842$ & 141922.4 & -263 & & 0858 & 43.61 & 13.30 & -21.48 \\
\hline 1RXS J142916.8 + 300520 & 142911.7 & 300438 & $\mathrm{MCG}+05$ & $0.052 \pm 0.0132$ & 41.89 & 15.50 & -18.38 \\
\hline 1RXS J143207.9 + 313504 & 143209.0 & 313505 & CGCG 1 & $0.079 \pm 0.0154$ & 43.22 & 15.50 & -21.26 \\
\hline 1RXS J143450.7 + 033834 & 143450.6 & 033843 & CGCG 047-107 & $0.056 \pm 0.0164$ & 41.95 & 15.30 & -20.04 \\
\hline 1RXS J151447.8 - 402157 & 151447.2 & -402135 & ESO 328-036-S & $0.162 \pm 0.0236$ & 42.79 & 15.85 & -19.08 \\
\hline 1RXS J151639.8 + 001454 & 151640.2 & 001502 & CGCG 021-063 & $0.078 \pm 0.0190$ & 43.16 & 15.60 & -21.02 \\
\hline 1RXS J151750.8 + 050615 & 151751.7 & 050628 & CGCG 049-106 & $0.107 \pm 0.0194$ & 43.03 & 15.60 & -20.38 \\
\hline 1 RXS J153210.0 + 585434 & 153216.1 & 585404 & VII Zw 608 & $0.028 \pm 0.0086$ & 42.90 & 17.60 & -19.49 \\
\hline 1RXS J153723.0 - 163551 & 153722.4 & -163545 & NGC 5959 & $0.054 \pm 0.0139$ & 42.34 & 14.50 & -20.47 \\
\hline 1RXS J153845.4 - 032309 & 153844.7 & -032248 & CGCG 022-021 & $0.070 \pm 0.0149$ & 42.44 & 15.29 & -19.65 \\
\hline 1RXS J155625.4 + 090311 & 155625.9 & 090319 & Mrk 863 & $0.115 \pm 0.0169$ & 43.15 & 15.30 & -20.88 \\
\hline 1RXS J161040.5 + 020626 & 161040.6 & 020639 & CGCG 023-021 & $0.105 \pm 0.0169$ & 42.76 & 15.40 & -19.91 \\
\hline 1RXS J161951.7 + 405834 & 161951.3 & 405847 & KUG $1618+410$ & $0.510 \pm 0.0289$ & 43.69 & 16.00 & -19.92 \\
\hline 1 RXS J162013.1 + 400858 & 162012.8 & 400906 & KUG 1618+402 & $0.356 \pm 0.0239$ & 43.28 & 16.00 & -19.30 \\
\hline 1RXS J162302.0 + 375506 & 162303.1 & 375521 & NGC 6137 & $0.087 \pm 0.0142$ & 42.06 & 13.40 & -22.10 \\
\hline 1RXS J162637.1 + 350239 & 162636.4 & 350242 & CGCG 196-064 & $0.081 \pm 0.0128$ & 42.81 & 15.70 & -20.02 \\
\hline 1RXS J162724.3 + 424041 & 162725.2 & 424047 & NGC 6159 & $0.108 \pm 0.0135$ & 42.86 & 15.20 & -20.32 \\
\hline 1RXS J162741.8 + 405536 & 162741.1 & 405537 & NGC 6160 & $0.069 \pm 0.0122$ & 42.68 & 14.20 & -21.36 \\
\hline 1RXS J170328.3 + 360400 & 170327.8 & 360420 & MCG +06-37-023 & $0.520 \pm 0.0095$ & 44.15 & 15.60 & -21.43 \\
\hline 1RXS J171227.2 + 355256 & 171228.4 & 355303 & MCG +06-38-005 & $0.125 \pm 0.0139$ & 42.79 & 15.00 & -20.16 \\
\hline 1RXS J171821.4 + 780118 & 171816.6 & 780105 & MCG +13-12-022 & $0.126 \pm 0.0102$ & 43.45 & 15.30 & -21.51 \\
\hline 1RXS J172215.7 + 304250 & 172215.4 & 304240 & MCG +05-41-010 & $0.077 \pm 0.0137$ & 43.06 & 15.60 & -20.78 \\
\hline
\end{tabular}


Table 2. continued.

\begin{tabular}{|c|c|c|c|c|c|c|c|}
\hline \multirow{3}{*}{$\begin{array}{l}\text { ROSAT-name } \\
\text { (1) }\end{array}$} & \multicolumn{2}{|c|}{ RA, Dec (J2000.0) } & \multirow{3}{*}{$\begin{array}{l}\text { Name } \\
\text { (4) }\end{array}$} & \multirow{3}{*}{$\begin{array}{c}\text { Countrate } \\
{\left[\mathrm{cts} \mathrm{s}^{-1}\right]} \\
(5)\end{array}$} & \multirow{3}{*}{$\begin{array}{r}\log \left(L_{X}\right) \\
{\left[\operatorname{ergs}^{-1}\right]} \\
(6)\end{array}$} & \multirow{3}{*}{$\begin{array}{r}m_{B} \\
{[\mathrm{mag}]} \\
(7)\end{array}$} & \multirow{3}{*}{$\begin{array}{r}M_{B} \\
\text { [mag] } \\
(8)\end{array}$} \\
\hline & [h m s] & {$[\mathrm{d} \mathrm{m} \mathrm{s}]$} & & & & & \\
\hline & (2) & (3) & & & & & \\
\hline 1RXS J172324.0 + 565840 & 172325.2 & 565828 & NGC 6370 & $0.054 \pm 0.0079$ & 42.45 & 13.88 & -21.36 \\
\hline 1RXS J173030.1 + 742244 & 173036.9 & 742234 & NGC 6414 & $0.072 \pm 0.0070$ & 42.95 & 15.40 & -20.75 \\
\hline 1RXS J180242.4 + 424734 & 180239.8 & 424746 & MCG +07-37-018 (NE) & $0.082 \pm 0.0114$ & 43.16 & 14.40 & -22.16 \\
\hline 1RXS J180634.6 + 613554 & 180635.6 & 613538 & MCG +10-26-015 & $0.091 \pm 0.0054$ & 42.66 & 16.20 & -18.98 \\
\hline 1RXS J182715.1 + 195624 & 182714.8 & 195619 & MCG +03-47-002 & $0.129 \pm 0.0179$ & 43.22 & 15.30 & -20.77 \\
\hline 1RXS J184038.1 - 770930 & 184038.5 & -770929 & ESO 045-011 & $0.449 \pm 0.0508$ & 42.98 & 13.70 & -20.59 \\
\hline 1RXS J190937.0 - 622853 & 190933.3 & -622840 & ESO 104-041 & $0.241 \pm 0.0467$ & 44.07 & 17.46 & -20.23 \\
\hline 1RXS J192700.9 - 534241 & 192701.6 & -534252 & ESO 184-068 & $0.309 \pm 0.0811$ & 43.85 & 15.99 & -20.88 \\
\hline 1RXS J193139.0 - 335426 & 193138.6 & -335443 & PKS 1928-34 & $0.254 \pm 0.0290$ & 44.23 & 17.00 & -21.02 \\
\hline 1RXS J200052.6 - 343808 & 200046.6 & -343800 & ESO 399-015 & $0.066 \pm 0.1920$ & 42.47 & 15.02 & -20.06 \\
\hline 1RXS J200606.1 - 542212 & 200906.4 & -542248 & SGC 2005.13-5431.6 & $0.157 \pm 0.0313$ & 43.48 & 14.20 & -22.47 \\
\hline 1RXS J201731.2 - 411452 & 201731.2 & -411452 & RXS J201731.2-411452 & $0.145 \pm 0.0390$ & & 17.00 & \\
\hline 1RXS J202304.1 + 093233 & 202304.3 & 093238 & CGCG 399-005 & $0.123 \pm 0.0167$ & 42.67 & 15.70 & -19.21 \\
\hline 1RXS J203433.1 - 303731 & 203431.4 & -303729 & IRAS F20315-3047 & $0.167 \pm 0.0351$ & 42.61 & 13.30 & -21.13 \\
\hline 1RXS J205522.8 + 022118 & 205522.3 & 022116 & CGCG 374-029 & $0.055 \pm 0.0118$ & 41.81 & 15.10 & -18.55 \\
\hline 1RXS J210221.7 + 105812 & 210221.6 & 105816 & CGCG 425-034 & $0.203 \pm 0.0211$ & 43.04 & 15.40 & -19.91 \\
\hline 1RXS J212324.3 + 021137 & 212324.3 & 021132 & CGCG 375-033 & $0.071 \pm 0.0156$ & 43.04 & 14.90 & -21.56 \\
\hline 1RXS J212541.2 - 490935 & 212540.7 & -490938 & Fairall 969 & $0.145 \pm 0.0237$ & 43.73 & 15.71 & -21.68 \\
\hline 1RXS J213833.0 + 320507 & 213933.4 & 320506 & CGCG 493-002 & $0.185 \pm 0.0217$ & 42.93 & 15.50 & -19.48 \\
\hline 1RXS J214153.6 + 315135 & 214153.5 & 315128 & CGCG 493-004 & $0.074 \pm 0.0149$ & 43.04 & 15.70 & -20.52 \\
\hline 1RXS J215213.9 - 194256 & 215209.6 & -194324 & ESO 600-014 & $0.141 \pm 0.0359$ & 43.94 & 15.90 & -22.04 \\
\hline 1RXS J215656.8 - 113920 & 215656.5 & -113932 & NGC 7158 & $0.285 \pm 0.0310$ & 43.38 & 17.30 & -17.93 \\
\hline 1RXS J221231.8 + 384049 & 221231.6 & 384058 & UGC 11950 & $0.072 \pm 0.0123$ & 42.36 & 13.90 & -20.68 \\
\hline 1RXS J221918.8 + 120757 & 221918.5 & 120753 & II Zw 177 & $0.247 \pm 0.0316$ & 44.05 & 17.00 & -20.61 \\
\hline 1RXS J222706.1 + 362140 & 222705.8 & 362142 & UGC 12040 & $0.058 \pm 0.0113$ & 42.30 & 14.30 & -20.36 \\
\hline 1RXS J223656.5 - 221321 & 223655.9 & -221312 & ESO 602-031 & $0.422 \pm 0.0430$ & 42.79 & 14.30 & -21.32 \\
\hline 1RXS J224156.7 - 423550 & 224152.8 & -423535 & ESO 290-003 (NE) & $0.090 \pm 0.0225$ & 42.84 & 15.64 & -20.04 \\
\hline 1RXS J225850.4 + 405610 & 225855.5 & 405553 & UGC 12282 & $0.065 \pm 0.0128$ & 42.17 & 14.49 & -19.73 \\
\hline 1RXS J230921.5 + 004540 & 230920.3 & 004523 & IC 5287 & $0.091 \pm 0.0180$ & 43.05 & 14.80 & -20.79 \\
\hline 1RXS J231357.7 - 113027 & 231357.0 & -113019 & MCG -02-59-006 & $0.177 \pm 0.0260$ & 43.96 & 15.70 & -21.75 \\
\hline 1RXS J231853.1 - 010338 & 231853.7 & -010338 & UGC 12492 & $0.069 \pm 0.0170$ & 42.83 & 14.60 & -20.74 \\
\hline 1RXS J231906.5 - 420653 & 231906.7 & -420537 & MCG -07-47-032 & $0.120 \pm 0.0329$ & 43.39 & 15.00 & -21.75 \\
\hline 1RXS J232856.8 + 085346 & 232858.5 & 085439 & MCG +01-59-085 & $0.092 \pm 0.0155$ & 44.08 & 18.00 & -20.75 \\
\hline 1RXS J233355.5 - 234336 & 233355.2 & -234341 & PKS 2331-240 & $0.437 \pm 0.0387$ & 43.81 & 16.50 & -19.90 \\
\hline 1RXS J234547.5 - 293053 & 234547.6 & -293104 & NGC 7749 & $0.079 \pm 0.0187$ & 42.80 & 13.82 & -21.92 \\
\hline 1RXS J235038.6 + 243321 & 235036.6 & 243322 & UGC 12804 & $0.051 \pm 0.0127$ & 42.62 & 15.11 & -20.58 \\
\hline 1RXS J235728.4 - 302739 & 235727.3 & -302737 & AM 2354-304-E & $0.057 \pm 0.0447$ & 42.55 & 15.82 & -19.63 \\
\hline \multicolumn{8}{|c|}{ Companion galaxies, galaxy pairs } \\
\hline 1RXS J023513.9 - 293616 & 023454.5 & -293428 & RX J023454.8-293425 & $0.006 \pm 0.0020$ & 45.00 & 17.90 & -24.53 \\
\hline 1RXS J043520.2 - 780150 & 043516.2 & -780157 & ESO 015-011 (b) & $0.077 \pm 0.004$ & & 14.20 & -22.78 \\
\hline 1RXS J071204.2 - 603005 & 071203.2 & -603030 & ESO 122-016 (SW) & \pm & & 14.60 & -20.99 \\
\hline 1RXS J083539.1 - 040508 & 083548.6 & -040533 & MCG -01-22-27 & \pm & & 15.00 & -18.93 \\
\hline 1RXS J085001.4 + 701804 & 084958.4 & 701758 & NGC 2650 & \pm & & 14.30 & -19.53 \\
\hline 1RXS J091345.4 + 474208 & 091344.7 & 474218 & MCG +08-17-60 (N) & \pm & & 15.70 & -20.97 \\
\hline 1RXS J102403.1 + 062954 & 102359.8 & 062908 & CGCG 037-023 & \pm & & 15.60 & -20.84 \\
\hline 1RXS J132016.3 + 330828 & 132017.5 & 330845 & NGC 5098 (E) & \pm & & 15.50 & -20.35 \\
\hline 1RXS J153210.0 + 585434 & 153209.4 & 585420 & VII Zw 608 (W) & \pm & & 16.10 & -17.13 \\
\hline 1RXS J180242.4 + 424734 & 180239.4 & 424718 & $\mathrm{MCG}+07-37-018(\mathrm{SW})$ & \pm & & 15.40 & -20.99 \\
\hline
\end{tabular}

\section{Results}

The optical spectra of the X-ray counterparts (AGN candidates) of our first observing run are published in Paper I. All spectra taken during the remaining 4 observing runs are presented in Fig. 11.

We discriminated between the main X-ray counterpart and the galaxy companion when we observed more than one galaxy in the ROSAT error box.

The results derived from our optical spectroscopy are given in Table 3. We list the name of the object (1), the observing run (2), total exposure time used (3), observed redshift (4), flux ratios of $[\mathrm{O}$ III] $\lambda 5007 / \mathrm{H} \beta$ (5) and $[\mathrm{N} \mathrm{II}] \lambda 6583 / \mathrm{H} \alpha$ (6), Balmer decrement of the broad components (7), the width (FWHM) of the broad $\mathrm{H} \alpha$ component (8) and the derived nuclear activity class (9). The galaxies already presented in Paper I are marked [P1]. Those galaxies that since have been classified as an AGN are marked [V]. The detailed literature can be found in the Veron-Cetty Catalogue of AGN (2006).

\subsection{Redshift distribution}

The redshifts we derived from the object spectra are given in Table 3. The typical error is less than $160 \mathrm{~km} \mathrm{~s}^{-1}$. There is no redshift information for the BL Lac candidates.

Figure 2 shows the redshift distribution of our newly identified RASS AGN (dark columns). Most objects have redshifts 
Table 3. Optical line properties.

\begin{tabular}{|c|c|c|c|c|c|c|c|c|}
\hline Name & Obs. Run & $\begin{array}{r}\text { Exp. } \\
\text { [s] }\end{array}$ & $\begin{array}{r}c z \\
{\left[\mathrm{~km} \mathrm{~s}^{-1}\right]}\end{array}$ & {$[\mathrm{O}$ III $] / \mathrm{H} \beta$} & {$[\mathrm{N}$ II $] / \mathrm{H} \alpha$} & $\overline{\mathrm{H} \alpha} / \mathrm{H} \beta$ & $\begin{array}{r}F W H M_{\mathrm{H} \alpha, \mathrm{br}} \\
{\left[\mathrm{km} \mathrm{s}^{-1}\right]}\end{array}$ & AGN type \\
\hline ESO 409-003 & $97 / 07$ & 1200 & 8520 & 4.52 & 1.27 & (1) & & LINER \\
\hline UGC 32 & $97 / 08$ & 1200 & 22260 & 0.10 & 2.47 & & & LINER \\
\hline CGCG 433-025 [P1] & $96 / 11$ & 1800 & 13520 & 38.00 & & 2.29 & 8250 & Sy 1 \\
\hline NGC 57 & $97 / 08$ & 600 & 5580 & & & & & non-ac \\
\hline NGC 71 & $97 / 08$ & 960 & 6750 & 0.10 & 7.97 & & & Sy 2 \\
\hline NGC 70 & 97/08 & 960 & 7080 & 0.10 & 7.28 & & & LINER \\
\hline PKS 0018-19 & 97/07 & 2100 & 28590 & 25.22 & 1.54 & & 8160 & Sy 1.9 \\
\hline ESO 350-015 & 97/07 & 600 & 15060 & & & & & non-ac \\
\hline $\mathrm{HCG} 4 \mathrm{a} \quad[\mathrm{P} 1]$ & $96 / 11$ & 900 & 7900 & 8.20 & 1.70 & & 2100 & Sy 1.9 \\
\hline VIII Zw 36 [P1] & $96 / 11$ & 900 & 12390 & 13.40 & 0.90 & 3.62 & 1950 & Sy 1 \\
\hline MCG -03-03-017 & $97 / 07$ & 600 & 16170 & 0.10 & 2.47 & & & Sy 2 \\
\hline ESO 113-010 [P1] & $96 / 11$ & 900 & 7705 & 5.30 & 1.40 & & 2000 & Sy 2 \\
\hline UGC 716 [P1] & $96 / 11$ & 900 & 17710 & & & & & non-ac \\
\hline NGC 427 [P1] & $96 / 11$ & 2700 & 10035 & 0.10 & 1.80 & & 5500 & Sy 1 \\
\hline RX J011232.8-320140 [P1] & $96 / 11$ & 900 & & & & & & BL Lac \\
\hline ESO 244-017 [P1] & $96 / 11$ & 900 & 7045 & 7.10 & 1.50 & 2.20 & 3000 & Sy 1 \\
\hline KUG 0128+328 & $97 / 08$ & 900 & 21090 & 54.28 & 2.05 & 4.96 & 2710 & Sy 1 \\
\hline MCG -01-05-031 [P1] & $96 / 11$ & 900 & 5420 & 3.70 & 0.70 & & & Sy 2 \\
\hline ESO 080-005［P1] & $96 / 11$ & 1800 & 8080 & 13.00 & 0.80 & & 3450 & Sy 1.8 \\
\hline ESO 416-002 [P1] & $96 / 11$ & 1500 & 17710 & 22.10 & 2.60 & & 16000 & Sy 1.9 \\
\hline PHL 1389 [P1] & $96 / 11$ & 1200 & & & & & & BL Lac \\
\hline IC 1867 [P1] & $96 / 11$ & 600 & 7680 & 0.10 & 6.70 & & & LINER \\
\hline NGC 1217 [P1] & $96 / 11$ & 600 & 6155 & & & & & Sy 2 \\
\hline NGC 1218 [P1] & $96 / 11$ & 1500 & 8590 & 0.10 & 21.50 & & 2950 & Sy 1.9 \\
\hline ESO 548-081 [P1] & $96 / 11$ & 1500 & 4110 & 0.10 & 7.90 & & 4450 & Sy 1.9 \\
\hline AM 0426-625 [P1] & $96 / 11$ & 600 & 5490 & & & & & LINER \\
\hline ESO 015-011［P1] & $96 / 11$ & 900 & 18280 & 5.40 & 0.70 & 1.99 & 2750 & Sy 1.8 \\
\hline MCG -02-12-050 [P1] & $96 / 11$ & 1800 & 10730 & 38.10 & 1.10 & 2.52 & 5400 & Sy 1 \\
\hline UGC 3134 [P1] & $96 / 11$ & 900 & 8665 & 4.00 & 0.80 & & & Sy 2 \\
\hline MCG -01-13-025 [P1] & $96 / 11$ & 1200 & 4765 & 2.90 & 0.90 & 3.92 & 4500 & Sy 1 \\
\hline UGC 3194 & $00 / 02$ & 1800 & 8364 & & 0.42 & & 650 & LINER \\
\hline ESO 552-039 [P1] & $96 / 11$ & 1500 & 11870 & 18.40 & 1.60 & 2.22 & 4000 & Sy 1 \\
\hline NGC 1713 & $98 / 03$ & 1200 & 4491 & & & & & LINER \\
\hline CGCG 469-001 [V] & $98 / 03$ & 1200 & 5787 & 9.48 & 0.82 & 36.92 & 6460 & Sy 1.9 \\
\hline MCG -02-14-009 [P1] & $96 / 11$ & 900 & 8530 & 8.60 & 0.90 & 2.86 & 2850 & Sy 1 \\
\hline UGC 3355 & $98 / 03$ & 600 & 7810 & & & & & non-ac \\
\hline ESO 120-023 [P1] & $96 / 11$ & 600 & 11280 & & & & & non-ac \\
\hline ESO 254-017 [P1] & $96 / 11$ & 1500 & 8925 & 0.10 & 2.10 & & & LINER \\
\hline ESO 425-019 & $00 / 02$ & 1200 & 6740 & & & & & non-ac \\
\hline PMN J0623-6436 [P1] & $96 / 11$ & 1200 & 38640 & & & 2.72 & 2500 & Sy 1 \\
\hline PMN J0630-2406 [P1] & $96 / 11$ & 600 & & & & & & BL Lac \\
\hline ESO 490-026 [P1] & $96 / 11$ & 900 & 7450 & 16.40 & 1.40 & 2.14 & 4100 & Sy 1 \\
\hline NGC 2256 & $98 / 03$ & 1200 & 5145 & & & & & non-ac \\
\hline NGC 2258 & $98 / 03$ & 1200 & 4060 & & & & & LINER \\
\hline CGCG 085-010 & $98 / 03$ & 1200 & 18256 & 4.73 & 0.55 & 2.68 & 2060 & Sy 1.5 \\
\hline NGC 2329 & $98 / 03$ & 1200 & 5838 & & & & & LINER \\
\hline B2 $0708+32 b \quad[V]$ & $98 / 03$ & 1500 & 19890 & 1.83 & 1.59 & 9.28 & 3880 & Sy 1.9 \\
\hline ESO 122-016 (NE) [P1] & $96 / 11$ & 1200 & 9770 & & & & & LINER \\
\hline UGC 3901 & $98 / 03$ & 1200 & 6508 & 0.65 & 0.78 & 6.43 & 9150 & Sy 1.9 \\
\hline UGC 3927 [V] & $98 / 03$ & 1200 & 12405 & & 4.98 & & 3850 & Sy 1.9 \\
\hline UGC 4018 & $00 / 02$ & 1200 & 8616 & & & & & LINER \\
\hline MCG +08-15-09 [V] & $98 / 03$ & 1200 & 7556 & 7.23 & 0.66 & 19.37 & 4970 & Sy 1.9 \\
\hline ESO 209-012 [P1] & $96 / 11$ & 1200 & 12140 & 8.60 & 1.60 & 2.93 & 3400 & Sy 1.5 \\
\hline CGCG 207-040 & $00 / 02$ & 1200 & 19249 & & & & & LINER \\
\hline MGC +08-15-56 [V] & $98 / 03$ & 1200 & 12583 & 3.97 & 0.94 & 6.07 & 1570 & NLS1 \\
\hline IC 505 & $98 / 03$ & 900 & 9821 & & & & 300: & LINER \\
\hline NGC $2617 \quad[\mathrm{~V}]$ & $98 / 03$ & 1200 & 4575 & 7.03 & 1.16 & 21.86 & 7408 & Sy 1.5 \\
\hline $\mathrm{MCG}+07-18-43$ & $98 / 03$ & 1200 & 16202 & & 2.16 & & 300: & LINER \\
\hline QSO east of NGC 2650 & $98 / 03$ & 420 & $(\mathrm{z}=1.90)$ & & & & & QSO \\
\hline CGCG 005-037 [V] & $00 / 02$ & 1500 & 17843 & 1.76 & 0.27 & 2.66 & 1440 & NLS1 \\
\hline MCG +08-17-60 [V] & $98 / 03$ & 1200 & 15953 & 8.40 & 0.07 & 10.18 & 3350 & Sy 1.5 \\
\hline
\end{tabular}


Table 3. continued.

\begin{tabular}{|c|c|c|c|c|c|c|c|c|}
\hline Name & Obs. Run & $\begin{array}{r}\text { Exp. } \\
\text { [s] } \\
(3)\end{array}$ & $\begin{array}{r}c z \\
{\left[\mathrm{~km} \mathrm{~s}^{-1}\right]} \\
(4)\end{array}$ & $\overline{[\mathrm{O}} \mathrm{III}] / \mathrm{H} \beta$ & $\overline{\overline{[\mathrm{N}} \mathrm{II}] / \mathrm{H} \alpha}$ & $\overline{\mathrm{H} \alpha / \mathrm{H} \beta}$ & $\begin{array}{r}F W H M_{\mathrm{H} \alpha, \mathrm{br}} \\
{\left[\mathrm{km} \mathrm{s}^{-1}\right]} \\
(8)\end{array}$ & AGN type \\
\hline $\begin{array}{ll}\text { VIII Zw } 45 \quad[\mathrm{~V}] \\
\end{array}$ & $98 / 03$ & 1200 & 12029 & 4.95 & 0.59 & 4.33 & 3480 & Sy 1.5 \\
\hline KUG $0929+540$ & $98 / 03$ & 1200 & 17340 & 0.94 & 0.06 & 2.07 & 1760 & NLS1 \\
\hline KUG 0933+511 [V] & $98 / 03$ & 600 & 16854 & 0.72 & 0.33 & 3.88 & 1820 & NLS1 \\
\hline CGCG 122-055 & $98 / 03$ & 1200 & 6769 & 14.25 & 1.17 & 3.05 & 3120 & Sy 1 \\
\hline NGC 3035 [V] & $98 / 03$ & 1200 & 4546 & & 1.92 & & 7460 & Sy 1.8 \\
\hline CGCG 064-024 & $98 / 03$ & 1200 & 23276 & & 4.64 & & 400: & LINER \\
\hline ESO 499-041 & $00 / 02$ & 1200 & 3826 & 1.80 & 0.58 & 5.26 & 1510 & NLS1 \\
\hline CGCG 037-022 & $98 / 03$ & 1200 & 13388 & 17.50 & 0.23 & 3.06 & 1940 & NLS1 \\
\hline CGCG 037-028 & $98 / 03$ & 1200 & 13204 & & 0.82 & & 500: & LINER \\
\hline CGCG 240-047 & $98 / 03$ & 1200 & 13191 & 16.83 & 0.80 & 7.52 & 2720 & Sy 1.5 \\
\hline GNB 069 [V] & $98 / 03$ & 1200 & 21690 & 1.54 & 1.13 & 1.99 & 3670 & Sy 1 \\
\hline CGCG 212-045 [V] & $98 / 03$ & 1200 & 11339 & 1.10 & 0.97 & & 3250 & Sy 1 \\
\hline $\mathrm{MCG}+07-23-15$ & $98 / 03$ & 1200 & 9394 & & & & & LINER \\
\hline CGCG 011-012 & $98 / 03$ & 1200 & 11724 & 9.96 & 0.89 & 6.80 & 3710 & Sy 1.5 \\
\hline NGC 3798 [V] & $98 / 03$ & 1200 & 3603 & 0.41 & 0.80 & 6.36 & 7990 & Sy 1.5 \\
\hline KUG $1141+371 \quad$ [V] & $98 / 03$ & 1200 & 11686 & 15.65 & 0.94 & 6.29 & 15310 & Sy 1 \\
\hline IC 734 & $00 / 02$ & 1800 & 23801 & & & & & non-ac \\
\hline MCG -01-30-041 [V] & $97 / 07$ & 1200 & 5700 & 3.00 & 0.64 & 2.66 & 5790 & Sy 1.8 \\
\hline VIII Zw 153 [V] & $98 / 03$ & 1200 & 36201 & & 0.19 & 2.23 & 4750 & Sy 1 \\
\hline ESO 267-013 & $97 / 07$ & 1200 & 4350 & 1.44 & 0.53 & & & LINER \\
\hline MCG +08-22-71 & $98 / 03$ & 1200 & 18841 & 4.48 & 0.68 & 4.37 & 2370 & Sy 1.8 \\
\hline Mrk 648 & $98 / 03$ & 1200 & 15534 & 0.69 & 0.00 & 3.84 & 2690 & NLS1 \\
\hline Mrk 202 [V] & $98 / 03$ & 1200 & 7234 & 3.15 & 0.37 & 2.87 & 2360 & NLS1 \\
\hline MCG +08-23-67 [V] & $98 / 03$ & 1200 & 9398 & 10.34 & 0.85 & 5.61 & 1510 & NLS1 \\
\hline ESO 381-007 [V] & $97 / 07$ & 1200 & 16470 & 9.85 & 0.98 & 3.50 & 4710 & Sy 1 \\
\hline KUG $1240+359$ [V] & $98 / 03$ & 1800 & 164591 & 0.40 & & & & QSO \\
\hline CGCG 015-026 [V] & $97 / 07$ & 1800 & 14190 & 19.86 & 2.13 & 2.80 & 5620 & Sy 1 \\
\hline NGC 4756 & $00 / 02$ & 1200 & 3981 & & & & & non-ac \\
\hline CGCG 043-056 & $97 / 07$ & 600 & 19650 & & & & & non-ac \\
\hline MCG -01-33-054 & $97 / 07$ & 1200 & 3960 & 0.10 & 1.60 & & & Sy 2 \\
\hline KUG 1256+241 [V] & $00 / 02$ & 1800 & 22586 & 8.12 & 0.97 & 1.87 & 5130 & Sy 1 \\
\hline CGCG 160-104 [V] & $98 / 03$ & 1200 & 7506 & -0.54 & -1.58 & & 690 & LINER \\
\hline IC $4165[\mathrm{~V}]$ & $98 / 03$ & 2400 & 8447 & & 1.02 & & 3810 & Sy 1.9 \\
\hline VIII Zw 272 & $98 / 03$ & 600 & 23513 & & & & & non-ac \\
\hline CGCG 160-193 [V] & $98 / 03$ & 1200 & 9870 & 0.58 & 0.97 & 1.91 & 3270 & Sy 1.5 \\
\hline NGC 5098 & $98 / 03$ & 600 & 10970 & & 3.76 & & 500: & LINER \\
\hline MCG +10-19-84 [V] & $98 / 03$ & 2400 & 35364 & 1.06 & 1.77 & 5.01 & 5610 & Sy 1 \\
\hline VIII Zw 327 & $98 / 03$ & 1200 & 27898 & 3.51 & & & & Sy 1.5 \\
\hline CGCG 365-016 & $00 / 02$ & 1200 & 13519 & 3.23 & 1.59 & 3.32 & 1100 & Sy 1.9 \\
\hline UGC 8829 [V] & $98 / 03$ & 1200 & 8015 & 5.66 & 1.15 & 9.35 & 19710 & Sy 1 \\
\hline CGCG 103-009 & $98 / 03$ & 1200 & 5000 & & 0.35 & & 230 & $\mathrm{H} \mathrm{II}$ \\
\hline $\mathrm{MCG}+05-33-24 \mathrm{a} \quad[\mathrm{V}]$ & $98 / 03$ & 1200 & 22705 & 1.53 & 0.46 & 3.89 & 1770 & Sy 1.8 \\
\hline CGCG 219-050 & $98 / 03$ & 1500 & 20656 & & 2.92 & & 7570 & Sy 1.5 \\
\hline NPM1G -16.0443 & $97 / 07$ & 1200 & 8730 & 5.24 & 0.80 & 6.16 & 2400 & Sy 1.5 \\
\hline CGCG 103-073 & $98 / 03$ & 600 & 7758 & & & & & non-ac \\
\hline CGCG 353-034 & $98 / 03$ & 600 & 13747 & & & & & non-ac \\
\hline ESO 511-030 [V] & $97 / 07$ & 600 & 6750 & 24.22 & 2.71 & 3.10 & 3620 & Sy 1 \\
\hline $\mathrm{MCG}+05-34-053$ & $97 / 08$ & 600 & 4470 & 0.10 & 0.52 & & & $\mathrm{H} \mathrm{II}$ \\
\hline CGCG 163-074 & $97 / 08$ & 1200 & 16620 & 8.64 & 0.68 & 2.80 & 3590 & Sy 1.5 \\
\hline CGCG 047-107 [V] & $98 / 03$ & 2700 & 8696 & 9.06 & 0.41 & 5.32 & 1320 & NLS1 \\
\hline ESO 328-036-S & $97 / 07$ & 1200 & 7230 & 0.10 & 1.93 & 2.86 & 3750 & Sy 1 \\
\hline CGCG 021-063 [V] & $97 / 07$ & 1800 & 15660 & 4.64 & 1.30 & & 3640 & LINER \\
\hline CGCG 049-106 & $97 / 07$ & 1200 & 11640 & 14.43 & 1.64 & 4.65 & 2190 & NLS1 \\
\hline VII Zw 608 & $97 / 08$ & 1200 & 19380 & 0.10 & 1.81 & & & LINER \\
\hline NGC 5959 & $97 / 07$ & 600 & 7350 & & & & & non-ac \\
\hline CGCG 022-021 [V] & $97 / 07$ & 1200 & 7260 & 3.90 & 0.98 & & 4310 & Sy 1.8 \\
\hline Mrk 863 [V] & $97 / 07$ & 600 & 12780 & 1.94 & 0.50 & 2.80 & 2760 & Sy 1.5 \\
\hline CGCG 023-021 & $97 / 07$ & 1800 & 8610 & 0.10 & 3.07 & & 4090 & Sy 1.9 \\
\hline KUG $1618+410 \quad$ [V] & $97 / 08$ & 1200 & 11370 & 14.64 & 0.27 & 2.78 & 2150 & NLS1 \\
\hline KUG $1618+402$ [V] & $97 / 08$ & 1200 & 8550 & 16.16 & 0.27 & 3.76 & 2160 & NLS1 \\
\hline NGC 6137 & $98 / 03$ & 600 & 9356 & & 1.03 & & 1440 & LINER \\
\hline CGCG 196-064 & $97 / 08$ & 1200 & 10350 & 1.25 & 0.25 & 4.06 & 1650 & Sy 1.9 \\
\hline NGC 6159 & $97 / 08$ & 600 & 9480 & & & & & LINER \\
\hline
\end{tabular}


Table 3. continued.

\begin{tabular}{|c|c|c|c|c|c|c|c|c|}
\hline Name & Obs. Run & $\begin{array}{l}\text { Exp. } \\
\text { [s] } \\
(3)\end{array}$ & $\begin{array}{r}c z \\
{\left[\mathrm{~km} \mathrm{~s}^{-1}\right]} \\
(4)\end{array}$ & $\begin{array}{r}{[\mathrm{O} \mathrm{III}] / \mathrm{H} \beta} \\
(5)\end{array}$ & {$[\mathrm{N}$ II $] / \mathrm{H} \alpha$} & $\begin{array}{r}\mathrm{H} \alpha / \mathrm{H} \beta \\
\text { (7) }\end{array}$ & $\begin{array}{r}F W H M_{\mathrm{H} \alpha, \mathrm{br}} \\
{\left[\mathrm{km} \mathrm{s}^{-1}\right]} \\
(8)\end{array}$ & AGN type \\
\hline NGC 6160 & $97 / 08$ & 600 & 9630 & & & & & non-ac \\
\hline MCG +06-37-023 & 97/08 & 600 & 18840 & & & & & LINER \\
\hline MCG +06-38-005 & 97/08 & 1200 & 8040 & 6.72 & 0.96 & 2.97 & 7450 & Sy 1.5 \\
\hline MCG +13-12-022 & 97/08 & 900 & 17010 & & & & & non-ac \\
\hline MCG +05-41-010 & 97/08 & 600 & 13980 & & & & & non-ac \\
\hline NGC 6370 & 97/08 & 600 & 8310 & & & & & LINER \\
\hline NGC 6414 & 97/08 & 1800 & 12600 & & & & & non-ac \\
\hline MCG +07-37-018 (NE) & $97 / 08$ & 600 & 15180 & & & & & non-ac \\
\hline MCG +10-26-015 & 97/08 & 600 & 8100 & & & & & non-ac \\
\hline MCG +03-47-002 & 97/08 & 1200 & 12180 & 4.38 & 1.02 & 2.80 & 3930 & Sy 1 \\
\hline ESO 045-011 & 97/07 & 1800 & 5370 & & & & & LINER \\
\hline ESO 104-041 (A) & 97/07 & 1200 & 25440 & & & 2.86 & 6980 & Sy 1 \\
\hline ESO $184-068$ & 97/07 & 600 & 17490 & & & & & non-ac \\
\hline PKS 1928-34 & 97/07 & 1800 & 29460 & 8.70 & 1.80 & & & Sy 2 \\
\hline ESO 399-015 & 97/07 & 1200 & 7740 & & & & & LINER \\
\hline SGC 2005.13-5431.6 & 97/07 & 600 & 16020 & & & & & non-ac \\
\hline RXS J201731.2-411452 & $97 / 07$ & 900 & & & & & & BL Lac \\
\hline CGCG 399-005 & 97/07 & 1200 & 7170 & 12.61 & 2.77 & & 6790 & Sy 1.9 \\
\hline IRAS F20315-3047 [V] & 97/07 & 1800 & 5760 & 6.02 & 1.21 & 2.92 & 3320 & Sy 1 \\
\hline CGCG 374-029 & 97/07 & 1200 & 4020 & 36.07 & 1.62 & 5.37 & 2040 & NLS1 \\
\hline CGCG 425-034 [V] & 97/07 & 2400 & 8610 & 1.60 & 0.44 & 2.80 & 6460 & Sy 1.5 \\
\hline CGCG 375-033 & 97/07 & 600 & 14520 & & & & & LINER \\
\hline Fairall 969 & 97/07 & 3600 & 22170 & 7.22 & 3.16 & 2.80 & 3660 & Sy 1 \\
\hline CGCG 493-002 & 97/08 & 1200 & 7380 & 4.84 & 0.69 & & 5220 & Sy 1.8 \\
\hline CGCG 493-004 & 97/08 & 1200 & 13050 & 0.41 & 0.04 & 2.84 & 1410 & NLS1 \\
\hline ESO 600-014 & 97/07 & 600 & 28470 & & & & & LINER \\
\hline NGC 7158 [P1] & $96 / 11$ & 900 & 8275 & 10.90 & 0.90 & & 2100 & NLS1 \\
\hline UGC 11950 & 97/08 & 600 & 6150 & & & & & LINER \\
\hline II Zw 177 [V] & 97/07 & 1800 & 24450 & 10.55 & 1.28 & 2.66 & 1180 & NLS1 \\
\hline UGC 12040 & 97/08 & 1800 & 6390 & 0.10 & 2.85 & & 4020 & Sy 1.9 \\
\hline ESO 602-031 [P1] & $96 / 11$ & 1800 & 9895 & 10.10 & 1.30 & & 5900 & Sy 1.8 \\
\hline ESO 290-003 (NE) & 97/07 & 1200 & 10170 & & & & & non-ac \\
\hline UGC 12282 & $97 / 08$ & 1200 & 5220 & 16.85 & 3.75 & & 5660 & Sy 1.9 \\
\hline IC 5287 & $96 / 11$ & 1800 & 9750 & 0.10 & 4.80 & 3.30 & 4200 & Sy 1 \\
\hline MCG -02-59-006 [P1] & $96 / 11$ & 900 & 22790 & 0.10 & 1.30 & & 3700 & Sy 1 \\
\hline UGC 12492 [P1] & $96 / 11$ & 600 & 8710 & & & & & LINER \\
\hline MCG -07-47-032 & $97 / 07$ & 600 & 16590 & & & & & non-ac \\
\hline MCG +01-59-085 & 97/07 & 1500 & 40860 & & & & & Sy 1 \\
\hline PKS 2331-240 [V] & 97/07 & 1800 & 14130 & 6.86 & & & 3700 & Sy 1.9 \\
\hline NGC 7749 & 97/07 & 600 & 10440 & & & & & LINER \\
\hline UGC 12804 & 97/08 & 600 & 10230 & & & & & non-ac \\
\hline AM 2354-304-E [V] & 97/07 & 1200 & 9150 & 0.10 & & 2.80 & 1970 & NLS1 \\
\hline \multicolumn{9}{|c|}{ Companion galaxies, galaxy pairs } \\
\hline RX J023454.8-293425 [P1] & $96 / 11$ & 3000 & 203550 & & & & & QSO \\
\hline ESO 015-011 (b) [P1] & $96 / 11$ & 900 & 18430 & 1.40 & 0.90 & & & LINER \\
\hline ESO 122-016 (SW) [P1] & $96 / 11$ & 1200 & 9780 & & & & & non-ac \\
\hline MCG -01-22-27 & $98 / 03$ & 1200 & 4575 & 7.03 & 1.16 & 21.86 & 7410 & H II \\
\hline NGC 2650 & $98 / 03$ & 1200 & 4356 & & 0.08 & & 8610 & Sy 1.8 \\
\hline MCG +08-17-60 (N) & 98/03 & 1200 & 15950 & & & & & non-ac \\
\hline CGCG 037-023 & $98 / 03$ & 1200 & 13158 & & 0.29 & & 410 & H II \\
\hline NGC $5098(\mathrm{E})$ & $98 / 03$ & 600 & 10970 & & & & & non-ac \\
\hline VII Zw 608 (W) & 97/08 & 1200 & 20640 & & & & & non-ac \\
\hline $\mathrm{MCG}+07-37-018(\mathrm{SW})$ & 97/08 & 900 & 14070 & 0.89 & 0.29 & & & H II \\
\hline
\end{tabular}

below $z=0.1$. Two objects not shown in Fig. 2 have redshifts of $z=0.55$ and $z=1.90$. Our median redshift is $z=0.0327$. The redshift distribution of the already known RASS AGN (Zimmermann et al. 2001) with a median redshift of $z=0.0330$ is shown for comparison (grey columns). The median redshift of all RASS AGN also amounts to $z=0.0330$.

These numbers can be compared with the SDSS redshift distribution of AGN found by Anderson et al. (2007). Limiting their $\mathrm{X}$-ray count rate to sources in excess of $0.05 \mathrm{cts} / \mathrm{s}$, they find a peak at $z \approx 0.15$. This shows that our ROSAT selected AGN are nearby objects compared to the Anderson sample.

\subsection{Optical and $X$-ray luminosity distribution}

The $B$-Band flux, the ROSAT soft (0.1-2.4 keV) X-ray flux as well as the luminosities of our sample galaxies are given in Table 2. A Hubble constant of $H_{0}=75 \mathrm{~km} \mathrm{~s}^{-1} \mathrm{Mpc}^{-1}$ was used throughout this paper. For all objects with redshift information 


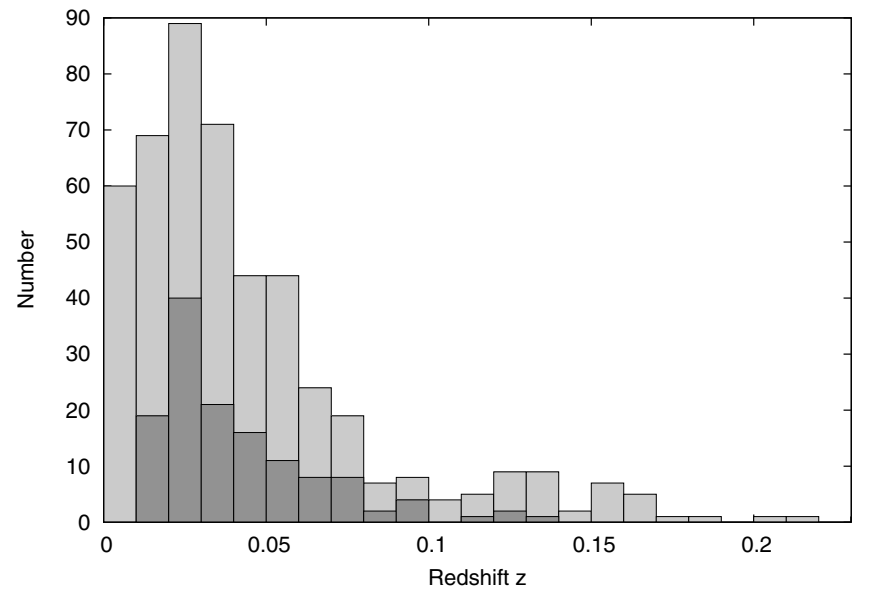

Fig. 2. Redshift distribution of our newly identified RASS AGN (dark columns). Two objects have redshifts of $z=0.55$ and $z=1.90$. The redshift distribution of the already known RASS AGN (Zimmermann et al. 2001) (grey columns) is shown for comparison.

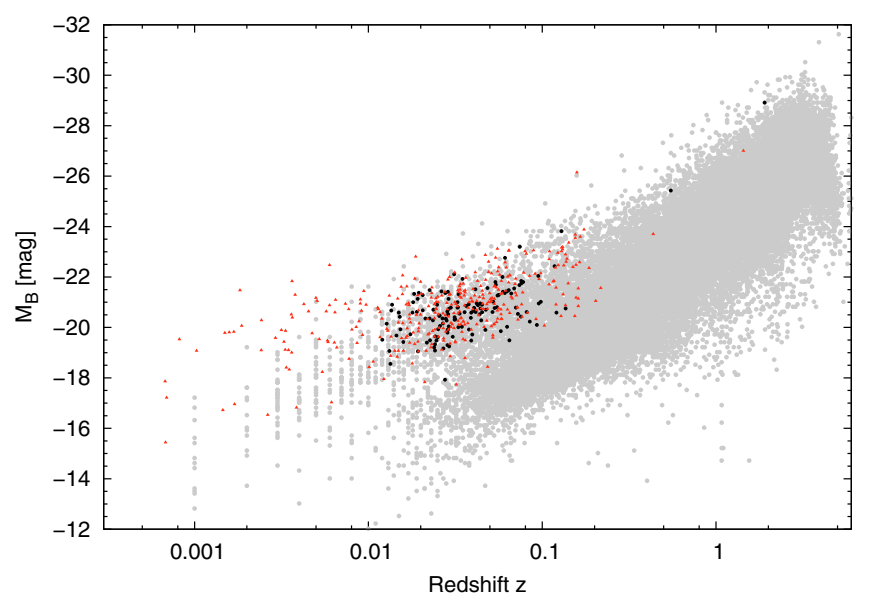

Fig. 3. $M_{B}$ distribution of our newly identified RASS AGN (black dots) as a function of redshift as well as the $M_{B}$ distribution of the already known RASS AGN (red dots). Grey points are objects from the 12th edition Quasar catalogue of Veron-Cetty (2006). We converted all magnitudes to a Hubble constant of $H_{0}=75 \mathrm{~km} \mathrm{~s}^{-1} \mathrm{Mpc}^{-1}$.

(all objects except those classified as BL Lac, see Table 3, Cols. 4 and 9) we calculated distances and luminosities. Four objects have absolute magnitudes $\leq-23$ mag classifying them as QSO.

The optical luminosity distribution of our sources is shown in Figs. 3 and 4. Optical magnitudes of our galaxies are taken from the PGC. We calculated in a homogeneous way the absolute $M_{B}$ magnitudes of our sample galaxies with the formula given by Veron-Cetty (2006) to compare our magnitudes with the AGN magnitudes in their Quasar and AGN catalogue. Figure 3 shows the $B$-band magnitudes $M_{B}$ of our newly identified RASS AGN (black dots) as a function of redshift, the $M_{B}$ distribution of the already known RASS AGN (red dots) as well as the Seyfert magnitude distribution in the Veron-Cetty Catalogue. The grey points represent all Seyfert/LINER galaxies given in the VeronCetty Catalog (2006). The absolute magnitudes from the VeronCetty Catalogue have been transformed to a Hubble constant of $H_{0}=75 \mathrm{~km} \mathrm{~s}^{-1} \mathrm{Mpc}^{-1}$.

The RASS selected AGN are located in optically bright galaxies compared to the AGN in the catalogue of

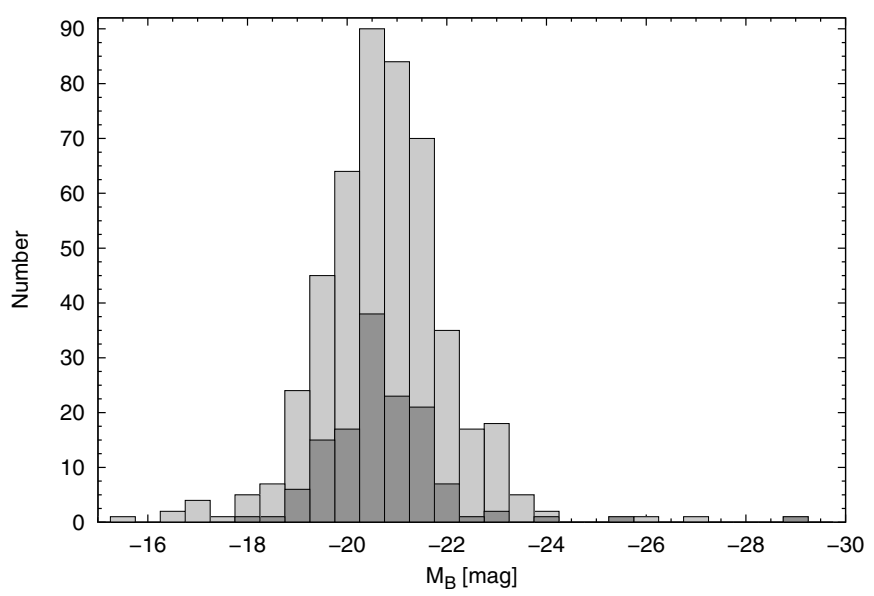

Fig. 4. $M_{B}$ distribution of our newly identified RASS AGN (dark columns) as well as the $M_{B}$ distribution of the already known RASS AGN (Zimmermann et al. 2001) (grey columns) shown for comparison.

Veron-Cetty (2006) (see Fig. 3). The mean absolute magnitude of our newly identified RASS AGN is $M_{B}=(-20.71 \pm$ $1.75)$ mag. This value is identical to the magnitude of the already known RASS AGN (Zimmermann et al. 2001) (see Fig. 4) and only slightly above the mean value $M_{B}=-20.46$ mag of Ho et al. (1997). Ho et al. (1997) derived their absolute magnitude from a sample of all nearby AGN. The optical brightness of our newly identified ROSAT AGN cannot be responsible for them remaining undetected so far.

To compute the soft X-ray $(0.1-2.4 \mathrm{keV})$ energy fluxes from the ROSAT count rates we assumed a power-law spectrum

$f_{E} \mathrm{~d} E \propto E^{-\Gamma+1} \mathrm{~d} E$,

where $f_{E} \mathrm{~d} E$ is the energy flux between $E$ and $E+\mathrm{d} E$. The spectral index was fixed to $\Gamma=2.3$, a typical value for galaxies observed with ROSAT (Hasinger 1991; Boller 1998). We only consider the Galactic H I-column density (Dickey 1990) along the line of sight as absorbing column density, so the given fluxes have to be understood as lower limits for the intrinsic fluxes emitted by the active nuclei from within its host galaxy.

In Fig. 5 we plot the X-ray luminosity of our newly identified RASS galaxies as a function of redshift $z$. Most objects lie one to two orders above the selection-limit of $0.05 \mathrm{cts} / \mathrm{s}$ indicated by the dotted line. The points below the selection limit are located at positions with a lower HI column density than the mean and therefore have lower flux levels than expected for objects with an average column density.

The X-ray luminosity distribution $L_{X}$ of our newly identified RASS AGN (dark columns) is shown in Fig. 6 together with the luminosity distribution $L_{X}$ of the already known RASS AGN (Zimmermann et al. 2001) (grey columns). The distribution of both samples is best described by a Gaussian with $F W H M=$ $1.66 \mathrm{dex}$ around $\log \left(L_{\mathrm{X}}\left[\mathrm{erg} \mathrm{s}^{-1}\right]\right)=42.9$. Zimmermann et al. (2001) obtained X-ray luminosities of $\log \left(L_{X}\right) \approx 43.5 \pm 1.5$ for their sample of active galaxies and $\log \left(L_{X}\right) \approx 42.5 \pm 1.5$ for the candidate galaxies (their Fig. 9a). They rated those X-ray sources as candidate galaxies that were likely to possess hitherto unreported active galactic nuclei. The X-ray luminosity distribution of X-ray AGN selected from the SDSS (Anderson et al. 2003, 2007) and having redshifts $z \leq 0.15$ is shown for comparison. Their mean X-ray $\operatorname{luminosity} \log \left(L_{\mathrm{X}}\right) \approx 43.5 \pm 1.1$ is slightly 


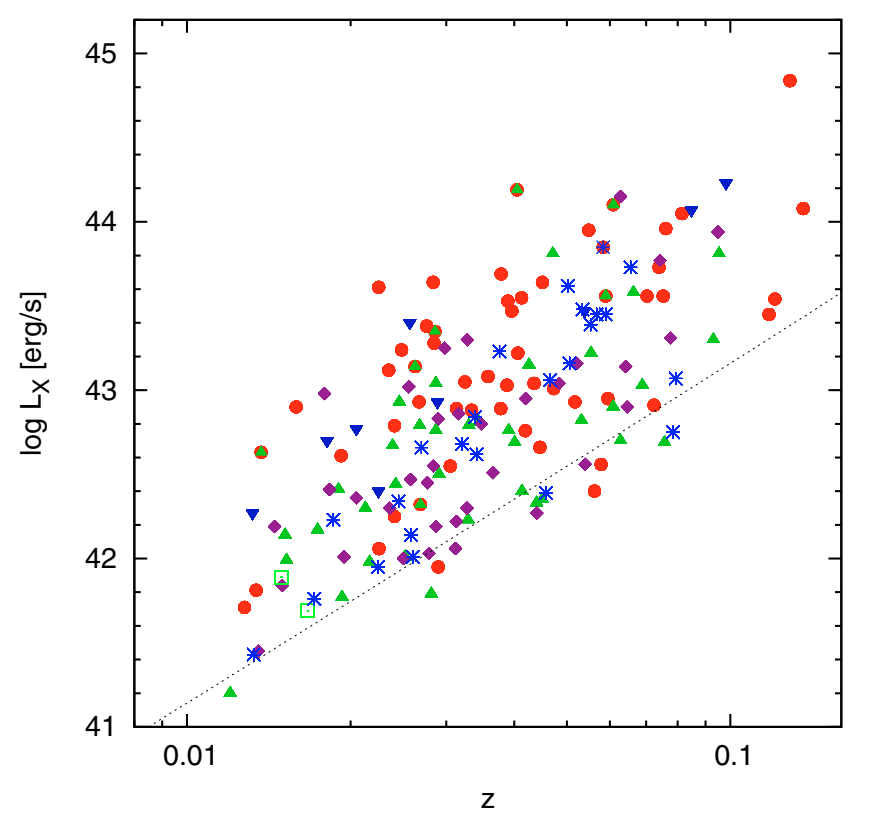

Fig. 5. X-ray luminosities as function of redshift $z$ of our newly identified RASS galaxies. Symbol definition as in Fig. 1. The dashed line marks the detection limit of the RASS bright source catalog of $0.05 \mathrm{cts} / \mathrm{s}$, converted to luminosities using the median galactic HI column density of our sample $\left(0.258 \times 10^{21} \mathrm{~cm}^{-2}\right)$.

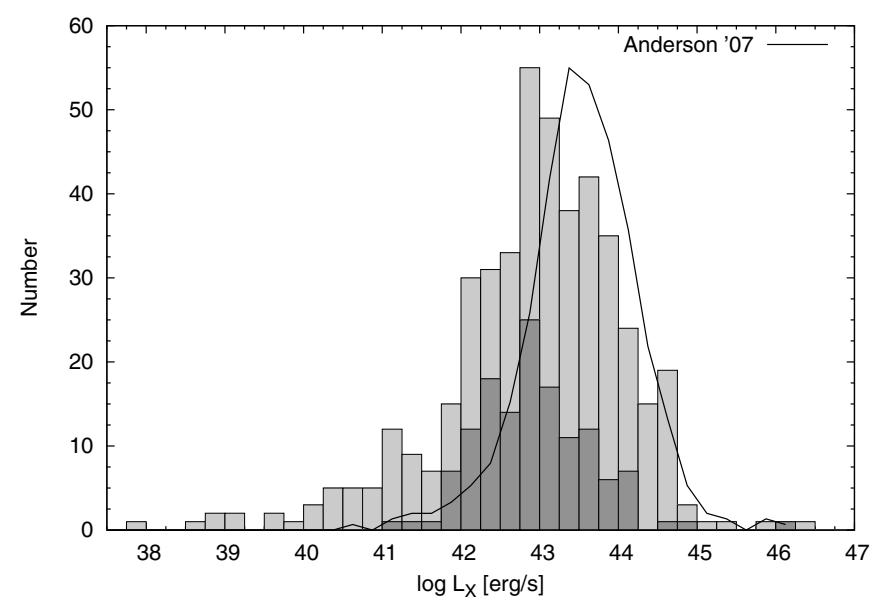

Fig. 6. Distribution of the X-ray luminosity $L_{\mathrm{X}}$ of our newly identified RASS AGN (dark columns) as well as the distribution of the already known RASS AGN (Zimmermann et al. 2001) (grey columns). Also shown for comparison is the relative distribution of Anderson et al. (2007) (solid line) for their X-ray AGN from the SDSS.

above our value. However, they identified mostly quasars and Seyfert 1 galaxies in their sample.

\subsection{AGN type distribution}

To classify the activity type of our galaxies we measured the $\mathrm{H} \alpha, \mathrm{H} \beta,[\mathrm{O} \mathrm{III}] \lambda 5007$, and [N II] $\lambda 6583$ emission line intensities whenever it was possible. The broad Balmer lines were fitted with one or multiple line components. Table 3 lists the measured line ratio of the $[\mathrm{O}$ III $] \lambda 5007 / \mathrm{H} \beta$ and $[\mathrm{N} \mathrm{II}] \lambda 6583 / \mathrm{H} \alpha$ line ratios. We considered only the narrow Balmer line components for these line ratios.

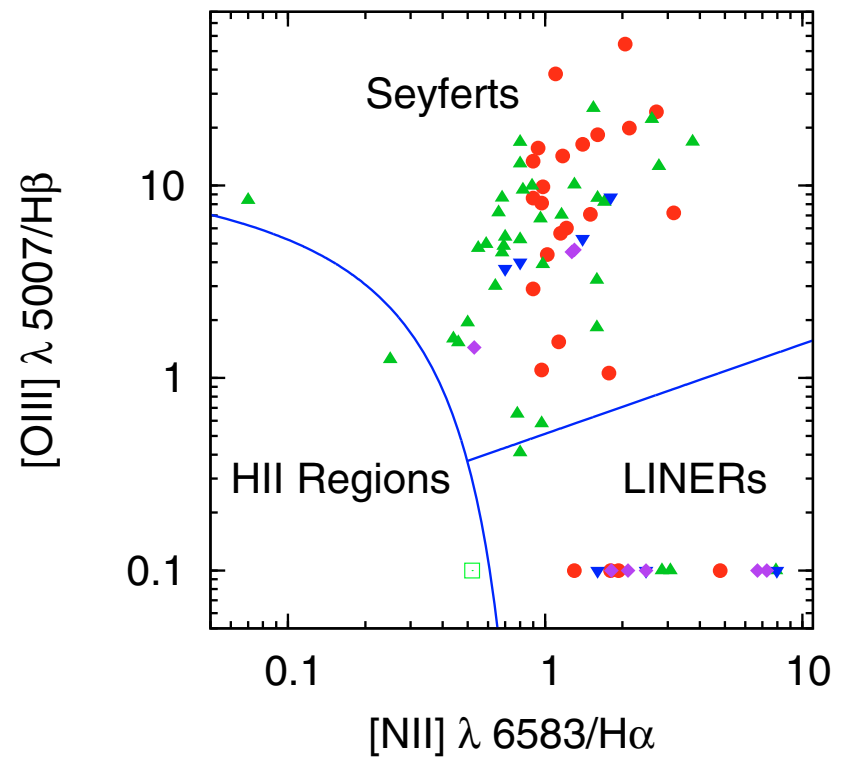

Fig. 7. Diagnostic Baldwin diagram of all our newly identified emission line galaxies with measured narrow components in all four lines. Also shown are the empirically determined dividing lines of Kewley et al. (2001) (solid line). Symbols are as described in Fig. 1.

The FWHM linewidth $W_{\alpha}$ of the broadest line component in the $\mathrm{H} \alpha$ complex is given in Table 3 (Col. 8). The typical error is $200 \mathrm{~km} \mathrm{~s}^{-1}$. Column 7 gives the Balmer decrement $\mathrm{H} \alpha / \mathrm{H} \beta$ derived from the broad-line components only.

We classified our galaxies in the following way: Seyfert 1 type galaxies have single broad Balmer line components only in their spectra. Intermediate Seyfert types 1.5, 1.8 and 1.9 show broad as well as narrow Balmer line components; the intensity ratio of the broad Balmer line component decreases with respect to the narrow Balmer line component. We recalibrated all our galaxies in a homogeneous way - including those of Paper I. In a few cases we made small changes in the classification of the intermediate type Seyferts. Narrow Line Seyfert 1 galaxies have single component Balmer lines with line widths of about less than $2500 \mathrm{~km} \mathrm{~s}^{-1}$ (Osterbrock 1985), only slightly broader than the forbidden narrow emission lines. Furthermore, these objects have relatively weak [O III] $\lambda 5007$ emission and strong FeII emission. Seyfert 2 galaxies, LINERs and HII galaxies show narrow emission lines only. On basis of their narrow line ratios [O III] $\lambda 5007 / \mathrm{H} \beta$ and $[\mathrm{N}$ II $] \lambda 6583 / \mathrm{H} \alpha$ we discriminate between Seyfert 2, LINER, and HII spectra (see Fig. 7). In Fig. 7 we show the dividing line for the different types (Kewley et al. 2001). In some LINERs we measured weak [N II] and $\mathrm{H} \alpha$ emission only, with upper limits for the [O III] $\lambda 5007$ line intensity. Here we set a value of 0.1 for the $[\mathrm{O} \mathrm{III}] \lambda 5007 / \mathrm{H} \beta$ intensity ratio.

BL Lac objects are characterized by their blue featureless continuum. Galaxies showing absorption line spectra only are classified as non-active galaxies.

Figure 8 shows the distribution of our newly identified RASS AGN and non-AGN types (dark columns) as well as the distribution of the already known RASS AGN (Zimmermann et al. 2001) (grey columns).

Only 27 galaxies (16.3\%) of our 166 ROSAT selected galaxies show no nuclear activity: 2 HII-galaxies (1.2\%) and 25 absorption line galaxies (15.1\%). Some of the X-ray counterparts we classified as absorption line galaxies have very high X-ray luminosities (see Fig. 5). Seven of them show $\log \left(L_{X}\right) \geq 43.45$. 


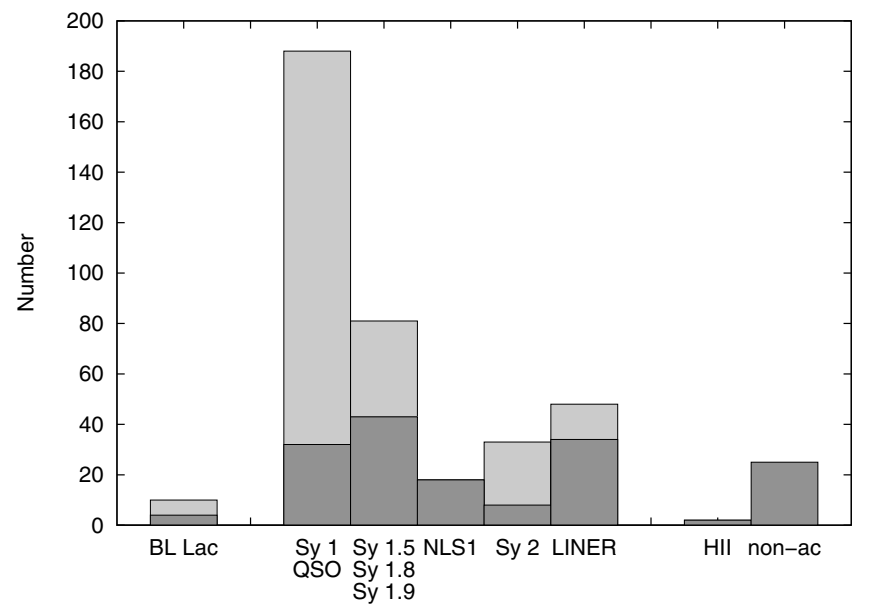

Fig. 8. Distribution of our newly identified RASS AGN and non-AGN types (dark columns) as well as the distribution of the already known RASS AGN (Zimmermann et al. 2001) (grey columns).

It is not clear whether we took spectra of the wrong counterpart or whether these objects are highly obscured AGN. ROSAT could also have detected X-ray emission from an unknown galaxy group or cluster of which our candidate galaxy may be a member.

Most of our galaxies (60.8\%) are of Seyfert type 1: 32 pure Seyfert 1 (19.3\%), 43 intermediate Seyfert 1 (25.9\%), and 18 NLS1 (10.8\%). About one fifth of the Seyfert 1 galaxies are NLS1 galaxies. Among all Seyfert galaxies only 8 galaxies $(4.8 \%)$ are of Seyfert type 2. Furthermore, we found 34 LINERs $(20.5 \%)$ and 4 BL Lac objects $(2.4 \%)$.

The AGN type distribution in our newly identified RASS AGN sample is similar to the distribution in the sample of Zimmermann et al. (2001) (see Fig. 8). However, the relative number of the pure Seyfert 1 types is greater in the sample of Zimmermann. On the other hand the relative number of intermediate Seyferts and LINERs is greater in our new RASS sample. This may be caused by the high quality of our spectra which allowed a more detailed classification.

\subsection{Companion galaxies and galaxy pairs}

The source of the X-ray emission could not always be identified with one optical galaxy only. In some cases we took more than one spectrum of objects within the X-ray error box.

We assigned that galaxy to the X-ray source that showed the highest activity degree in the optical spectrum. Data for the galaxies we identified as companion galaxies of the X-ray galaxies are given at the end of Table 2 and 3. MCG -01-22-27 is the companion of NGC 2617.

The X-ray quasar 1RXS J085001.4 + 701804 is an interesting object. This quasar shines through the outer disk of the spiral galaxy NGC 2650. We see absorption lines from the foreground galaxy in the quasar spectrum as a result of this projected superposition. pairs.

The rest of the new companion galaxies in our list are galaxy

\subsection{Line width distribution}

We derived $\mathrm{H} \alpha, \mathrm{H} \beta$, and [O III] $\lambda 5007$ line widths $(F W H M)$ of all emission line galaxies of our new sample from single or

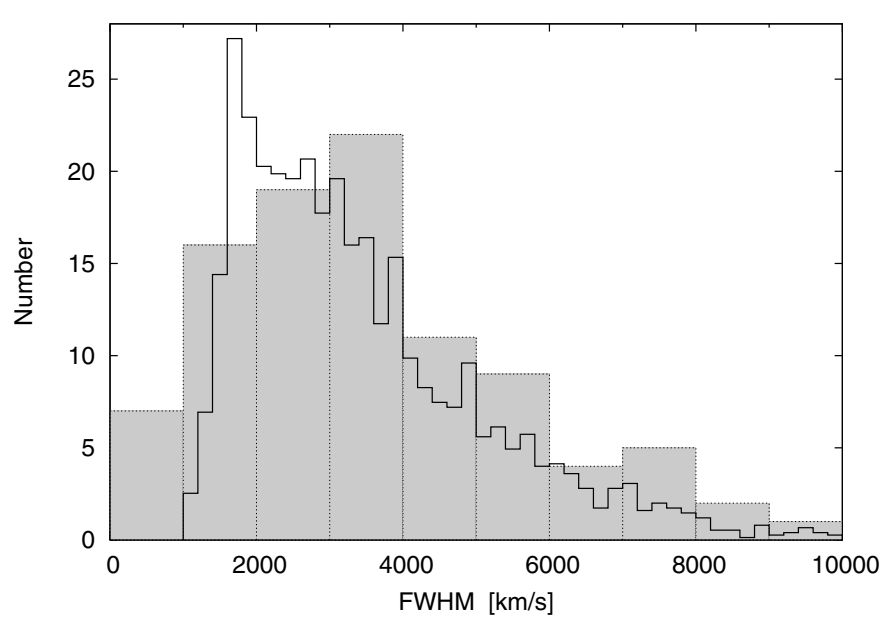

Fig. 9. $\mathrm{H} \alpha$ line width distribution $(F W H M)$ of our newly identified ROSAT AGN sample in steps of $1000 \mathrm{~km} \mathrm{~s}^{-1}$. The solid line gives the scaled-down distribution of $\mathrm{H} \alpha$ line widths of AGN derived from the Sloan Digital Sky Survey (Hao et al. 2005).

multi-component fits. The spectral lines were adapted by singlecomponent fits for starburst HII galaxies, LINERs, Seyfert 2 galaxies, Narrow-Line Seyfert 1 galaxies (NLS1), and pure Seyfert 1 galaxies. If the Balmer lines were fitted by more than one broad component and if the broadest component had a relative line intensity of at least 30 percent we present the line widths $(F W H M)$ of the broadest component in Table 3 (Col. 8) and Fig. 9.

We show the $\mathrm{H} \alpha$ line width distribution (FWHM) of our AGN sample in Fig. 9. A step size of $1000 \mathrm{~km} \mathrm{~s}^{-1}$ was used. For comparison we also plot (solid line) the relative distribution of the $\mathrm{H} \alpha$ line widths (FWHM) of Hao et al. (2005) who used a sample of AGN derived from the Sloan Digital Sky Survey (SDSS). The H $\alpha F W M$ distribution of our ROSAT AGN sample resembles the $\mathrm{H} \alpha F W H M$ distribution of the SDSS AGN sample.

\subsection{Optical to blue color distribution}

Finally, we inspected the optical colors of our galaxy spectra to check whether the hitherto unknown ROSAT selected AGN have specific continuum properties. We derived $B$ and $V$-band colors of our newly identified RASS-AGN with the IRAF task "sbands". Not all of our spectra cover the full wavelength range of the $B$-band filter. Therefore, we derived our $B$ and $V$-band colors with a bandpass width of $200 \AA$ only for the sake of homogeneity. We compared these "narrow-band" colors with "full bandwidth" colors whenever we had the full wavelength range at our disposal. Furthermore, we compared our "narrow-band" colors with data from the literature. They agree amongst each other within $0.1 \mathrm{mag}$.

We then compared the colors of our newly identified RASS AGN with an independent sample of all 764 AGN spectra derived from the Second Data Release of SDSS (Abazajian et al. 2004). These SDSS-AGN were selected by their classification as QSO in the SDSS database and limited to $z \leq 0.15$ to fit the redshift range of our sample. We determined the colors of these SDSS-AGN in the same way as explained above. The results are shown in Fig. 10.

The color distribution of the SDSS sample (black solid line) shows a Gaussian-like shape with a mean value of 


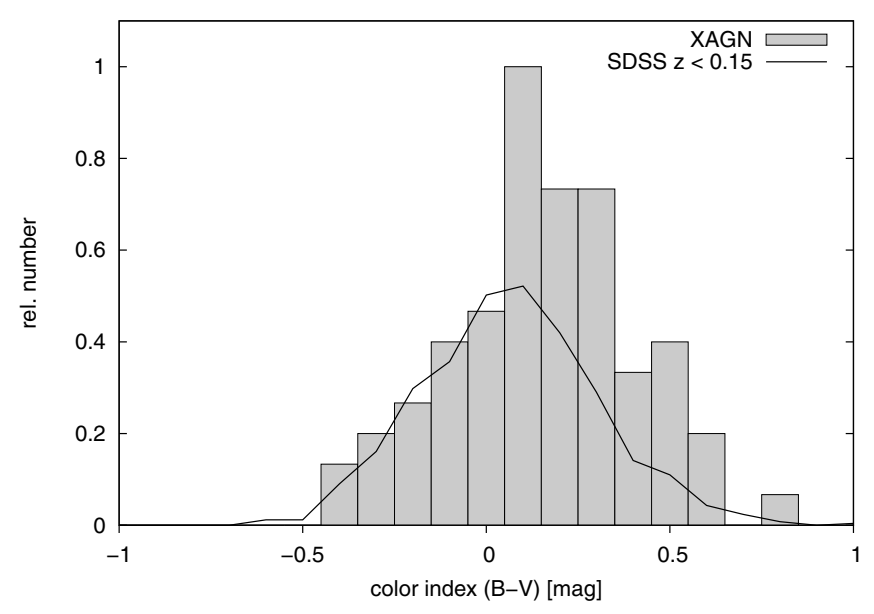

Fig. 10. Color distribution of our newly identified RASS AGN (shaded grey) and of a comparison sample consisting of all $764 z \leq 0.15$ AGN from the Second Data Release of SDSS (black line).

( $B-V \approx 0.05 \mathrm{mag}$ ). The distribution of our newly identified RASS AGN contains far more red galaxies.

The shift of the $(B-V)$ distribution to the red by about 0.15 mag might be caused by the fact that the newly identified RASS AGN are systematically redder and/or that the relative number of intermediate Seyfert galaxies and LINERs is higher compared to optically selected AGN samples. Intermediate Seyfert galaxies and LINERs have redder colors than pure Sy 1 types. We derived the following colors from the spectra of our individual AGN types: Sy 1: $0.04 \pm 0.16 \mathrm{mag}$, Sy $1.5-1.9$ : $0.13 \pm 0.26 \mathrm{mag}, \mathrm{NLS} 1:-0.09 \pm 0.21 \mathrm{mag}$, Sy $2: 0.28 \pm 0.27 \mathrm{mag}$, LINER: $0.34 \pm 0.16 \mathrm{mag}$.

\section{Discussion and conclusion}

About 550 compact X-ray sources have been identified as AGN or AGN candidates by cross-correlating the ROSAT Bright Source Catalog with the Principal Catalog of Galaxies. 350 of these sources have been identified before as AGN on basis of their optical spectra. Cross-correlating the ROSAT Bright Source Catalog with the Principal Catalog of Galaxies yielded a sample of 198 galaxies with as yet unknown X-ray emission.

We took spectra of as many X-ray counterparts as possible in five optical spectroscopic campaigns. At the end we obtained spectra of $166 \mathrm{X}$-ray counterparts. Since the start of our identification campaign, 41 of these galaxies have likewise been identified as AGN by other observers (see introduction). Our AGN classification of the different types agrees with the classification of other authors - except for minor discrepancies in the subdivision. For homogeneity we used our spectra and classification schema for this investigation.

Most of our galaxies are nearby objects. We determined redshifts up to 0.15 with a median of $z=0.033$. Only two AGN of our new sample are more distant objects (up to $z=1.9$ ).

The RASS selected AGN show a mean absolute magnitude of $M_{B}=(-20.71 \pm 1.75) \mathrm{mag}$ in the optical. They are hosted by brighter galaxies compared to the AGN in the catalogue of Veron-Cetty (2006) (see Fig. 3). Ho et al. (1997) derived a mean magnitude of $M_{B}=-20.46 \mathrm{mag}$ from a sample of all nearby AGN. Therefore it is not due to their optical faintness that our ROSAT selected AGN have not been detected before.
Our X-ray luminosity distribution of the RASS selected AGN peaks at $\log \left(L_{X}\left[\mathrm{erg} \mathrm{s}^{-1}\right]\right)=42.9 \pm 1.7$ (Fig. 6). This value should be compared with the peak in the luminosity $\log \left(L_{X}\right) \approx$ $43.5 \pm 1.1$ of X-ray AGN selected from the SDSS (Anderson et al. 2003, 2007) with redshifts below $z \leq 0.15$. They identified mostly quasars and Seyfert 1 galaxies in their sample which might have caused the peak at slightly higher luminosities in the luminosity distribution.

We determined the activity type of our emission line galaxies on the basis of their Balmer line widths as well as narrow emission line ratios. 139 galaxies of our 166 ROSAT selected objects have been classified as AGN. This indicates that the Xray selection criterion is very successful in finding new AGN. In the remaining 27 galaxies, faint AGN might still be buried that could not be separated in our spectra from the galaxy emission.

We compared the relative numbers of our Seyfert, LINER, $\mathrm{H} \mathrm{II}$ and absorption line galaxies with the numbers derived from the Palomar Survey of all nearby galaxies (Ho et al. 1997). While the fraction of galaxies showing no sign of nuclear activity is the same in both samples (XAGN: $15.1 \%$ vs. Ho: $13.6 \%$ ) we find far more Seyfert type nuclei (XAGN: $60.8 \%$ vs. Ho: $10.7 \%$ ) in our ROSAT selected AGN. Contrary to this, H II galaxies are found in nearly half of all Palomar galaxies (Ho: $42.4 \%$ vs. XAGN: $1.2 \%)$. The relative number of LINERs is higher in the Palomar Survey (XAGN: $20.5 \%$ vs. Ho: $32.7 \%$ ). This might be caused by the fact that it is more difficult to detect weak LINER properties in distant galaxies because of the lower $\mathrm{S} / \mathrm{N}$ ratio.

The fraction of our new ROSAT-selected Seyfert 1 galaxies (pure Sy1, intermediate Sy1, and NLS1) is very high compared to type 2 Seyferts (see Fig. 8). The Seyfert $1 / 2$ ratio is $11 / 1$. This ratio is strongly affected by selection effects as ROSAT was most sensitive in the soft X-ray band which is heavily absorbed in type 2 AGN. Optical surveys do not suffer from this bias. Therefore, an Sy1/Sy2 ratio of 1:1.4 or an Sy 1 fraction of $42 \%$ as reported by Ho et al. (1997) may better reflect the true ratio.

We classified 18 galaxies as Narrow Line Seyfert 1 which is $20 \%$ of all broad-line AGN (Sy1 to Sy1.9). Other studies of RASS selected sources also detected many Narrow Line Seyfert 1 galaxies (e.g. Boller et al. 1996; Grupe et al. 1999). From NLS1 catalogs compiled from the SDSS AGN Catalog, Williams et al. (2002) found that roughly $15 \%$ of all broad line AGN are of NLS1-type, a number already suggested by Osterbrock (1987) and later confirmed by Zhou et al. (2007), who determined an average fraction of $14 \%$ (up to $\approx 20 \%$ at $M_{\mathrm{g}} \approx-22 \mathrm{mag}$ ) for their sample of $>2000$ NLS1-galaxies from SDSS-DR3.

The linewidth distribution of our ROSAT sample matches that of the SDSS AGN (Hao et al. 2005).

We also determined optical broad-band colors from the galaxy spectra. We found that our hitherto undetected ROSAT AGN have redder colors in comparison to an independent sample of AGN spectra derived from the Second Data Release of SDSS (Abazajian et al. 2004) (see Fig. 10).

The redder color of our newly identified RASS AGN might explain why they have not been detected in earlier AGN searches which concentrated on blue or UV-excess galaxies or on optical identifications based on low resolution objective prism spectra. All these surveys were not complete and were biased towards single broad band or spectral properties. That our galaxies predominantly are fainter in the blue might be caused by internal absorption of the central nonthermal radiation within the host galaxy. Another explanation could be that the redder distribution of our newly identified RASS AGN might be caused by the 

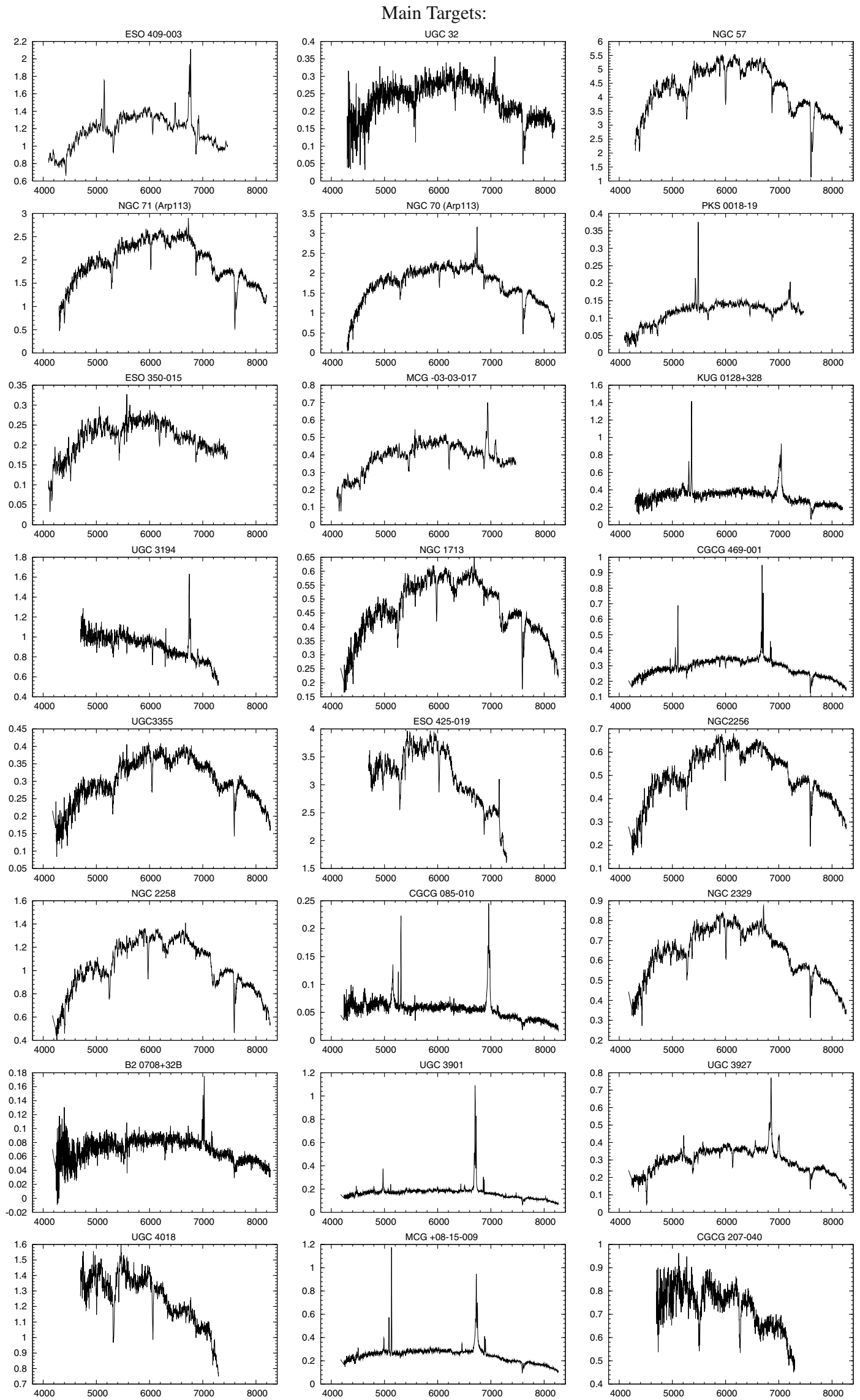

Fig. 11. Spectra of all objects of our sample. 

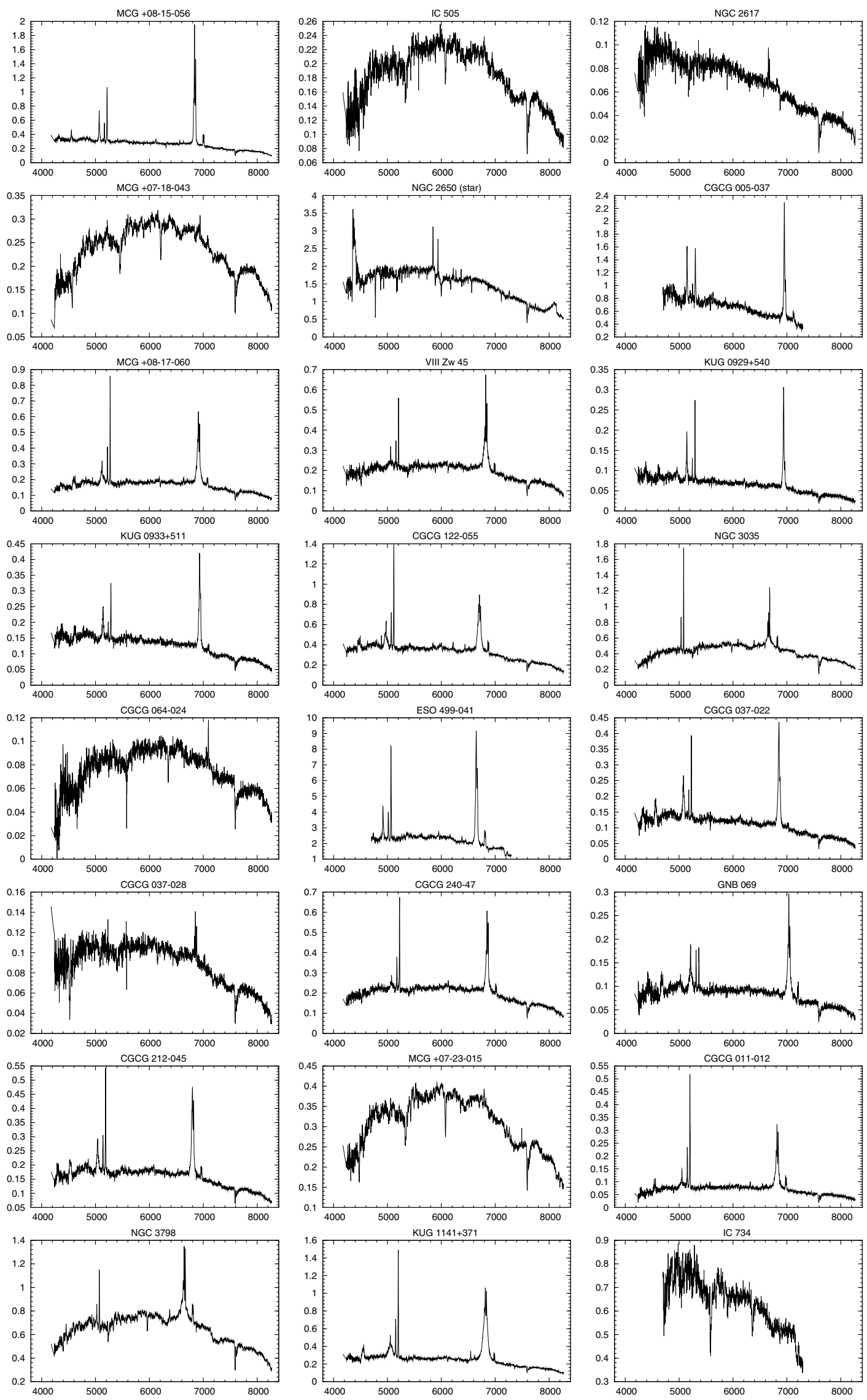

Fig. 11. continued. 

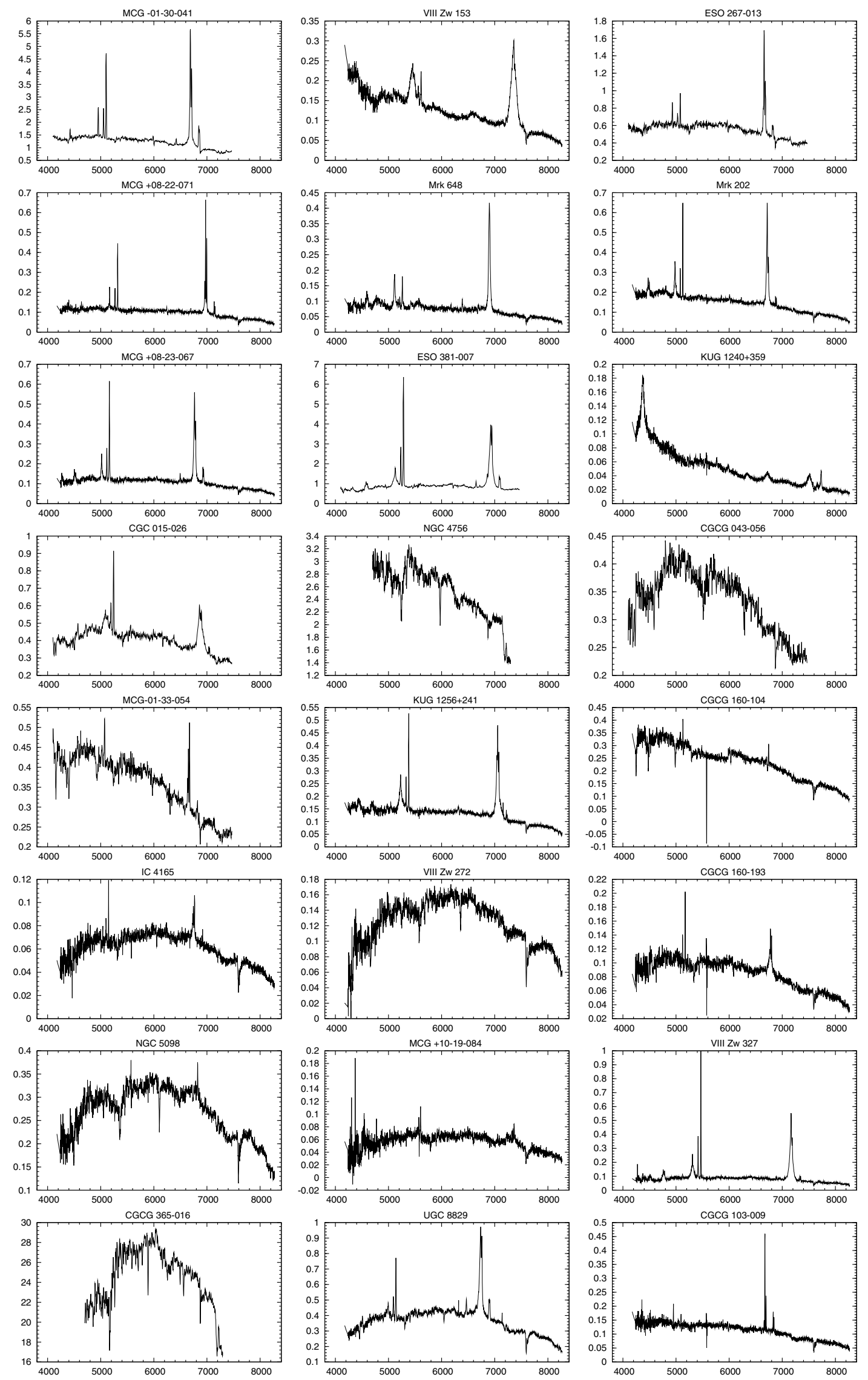

Fig. 11. continued. 

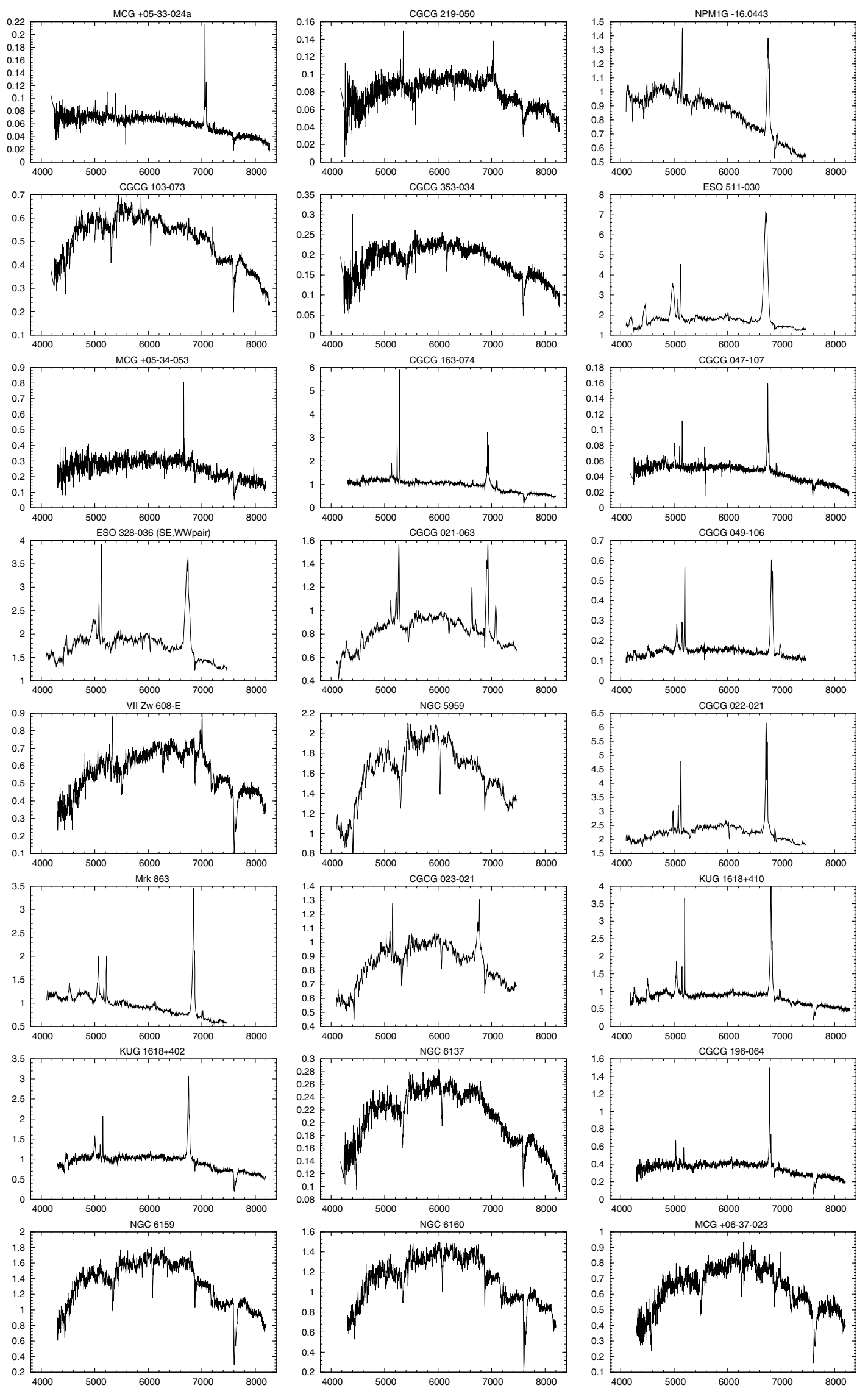

Fig. 11. continued. 

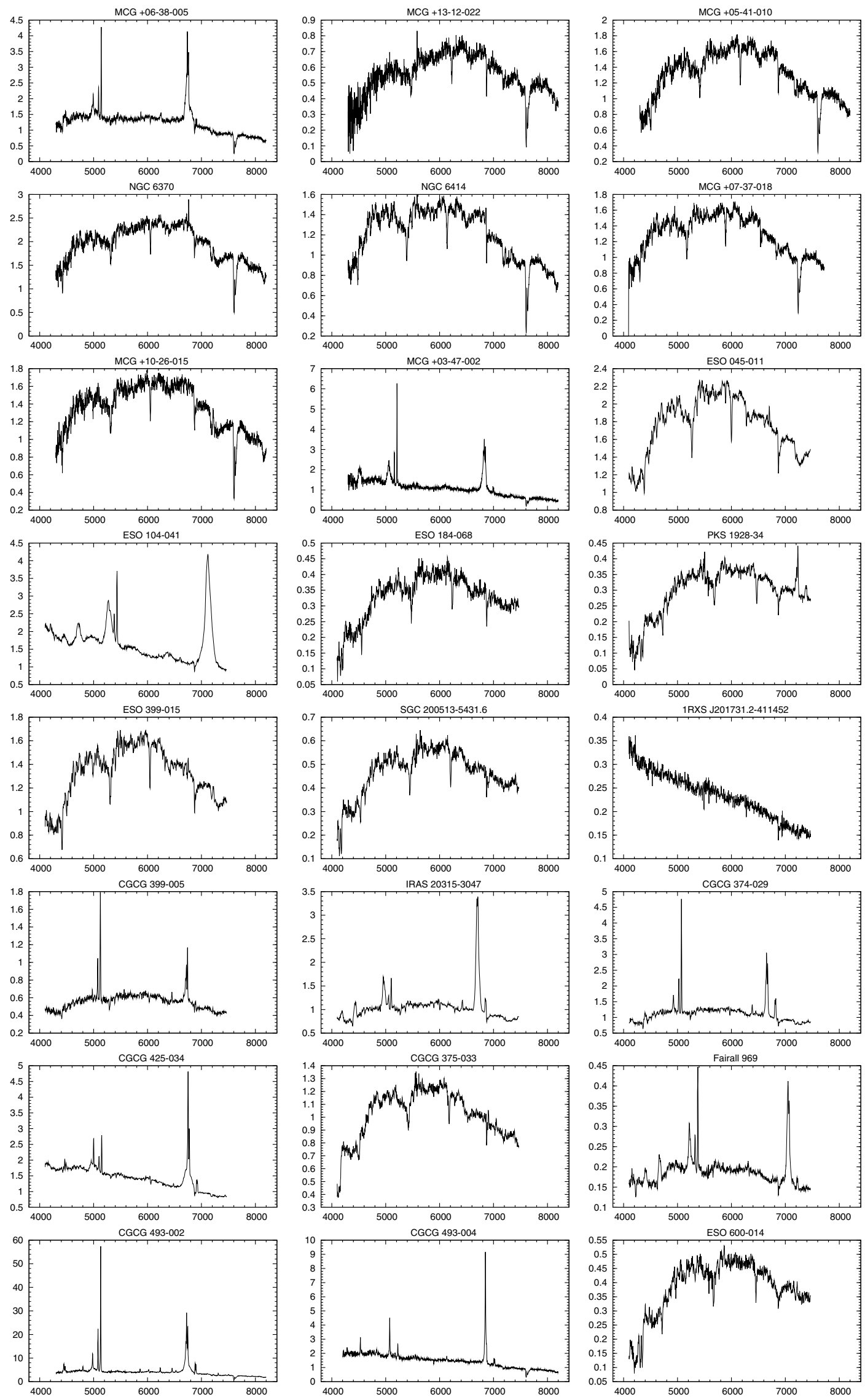

Fig. 11. continued. 

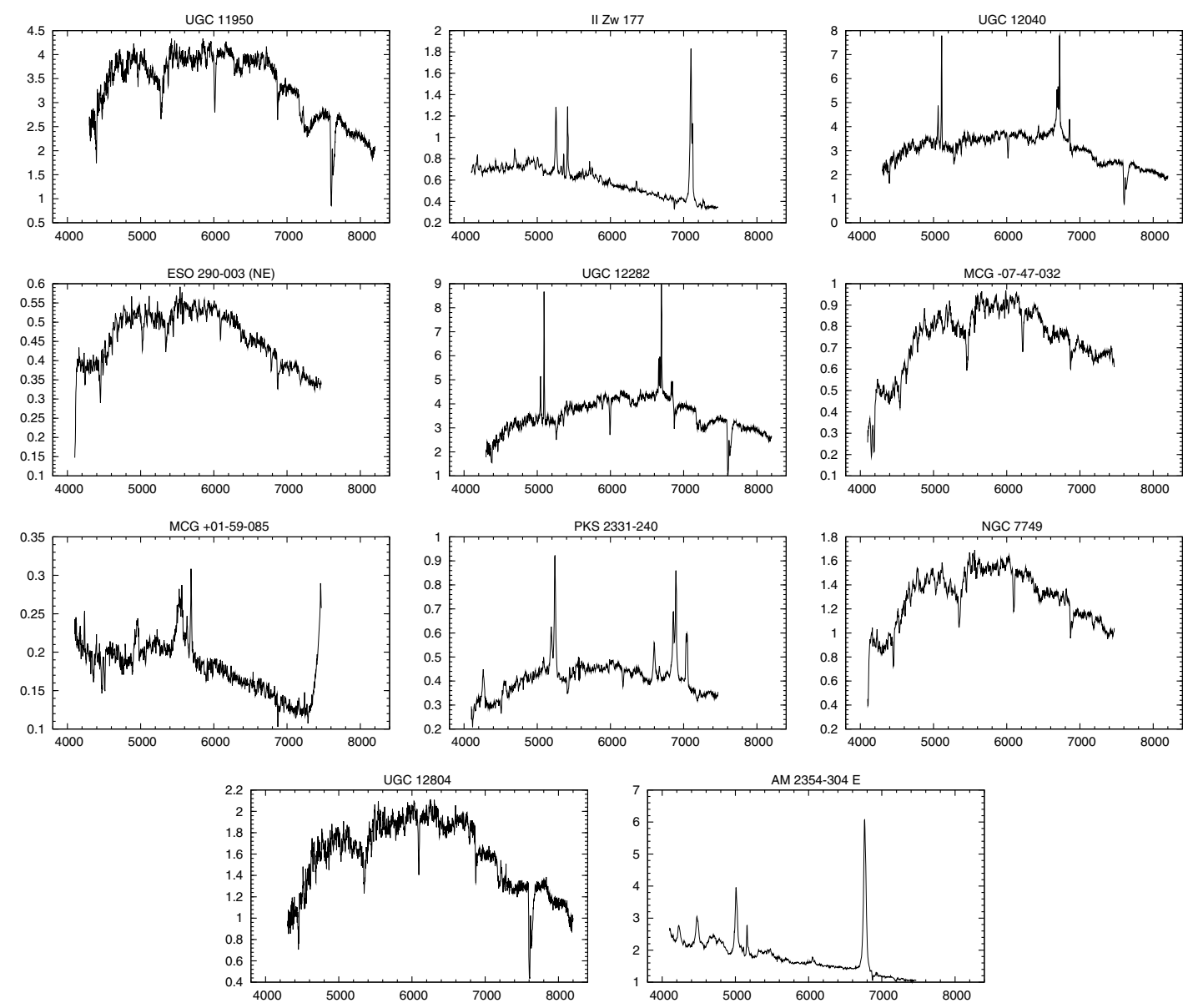

Fig. 11. continued.
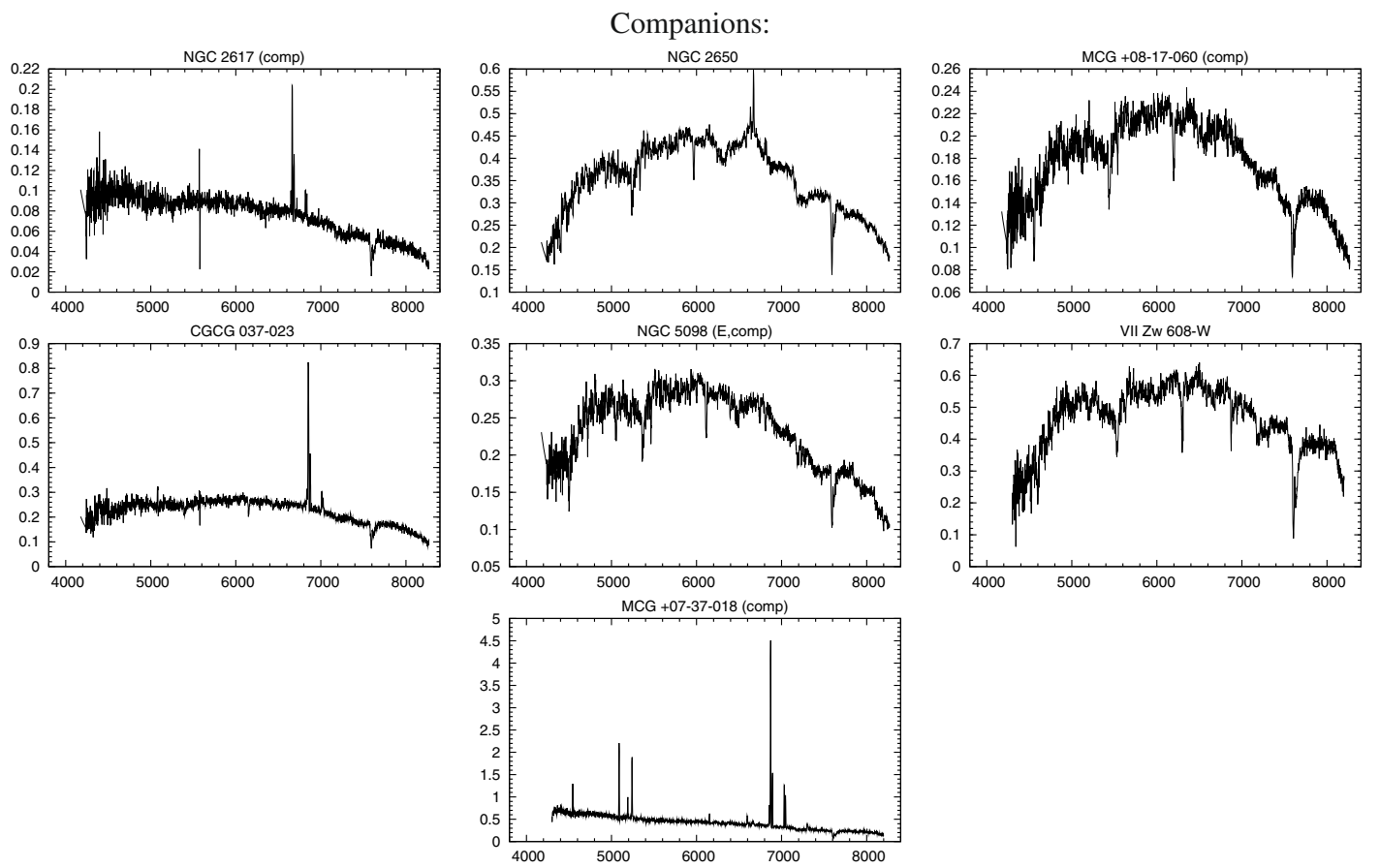

Fig. 11. continued. 
fact that the relative number of intermediate Seyfert galaxies and Liners in our sample is higher compared to other optically selected AGN samples. Intermediate Seyfert galaxies and Liners have redder colors than pure Sy 1 types.

In a future paper we will supplement our optical and X-ray data with data at radio and far-infrared wavelengths to further constrain our classification and to gain a deeper insight into their $\mathrm{X}$-ray production mechanisms.

Acknowledgements. The ROSAT project was supported by the German Bundesministerium für Bildung, Wissenschaft, Forschung und Technologie (BMBF/DARA) and by the Max Planck Gesellschaft (MPG).

This work was partially supported by the Deutsche Forschungsgemeinschaft, $D F G$ project number Ko 857 and the "Verbundforschung Astrophysik" (Grant 05 AE5PD 1/4).

\section{References}

Abazajian, K., Adelman-McCarthy, J. K., Agüeros, M. A., et al. 2004, AJ, 128, 502

Adelman-McCarthy, J., Agueros, M. A., Allam, S. S., et al. 2006, ApJS 162, 38 Anderson, S. F., Voges, W, Margon, B., et al. 2003, AJ, 126, 2209

Anderson, S. F., Margon, B., Voges, W., et al. 2007, AJ, 133, 313

Appenzeller, I., Thiering, I., Zickgraf, F. J. et al. 1998, ApJS 117, 319

Bade, N., Engels, D., Voges, W., et al. 1998, A\&AS, 127, 145

Bauer, F. E., Condon, J. J., Thuan, T. X., \& Broderick, J. J. 2000, ApJS, 129, 547

Bischoff, K., Pietsch, W., Boller, T., et al. 1999, MPE Rep., 272, 226

Boller, T., Brandt, W. N., \& Fink, H. 1996, A\&A, 305, 53
Boller, T., Bertoldi, F., Dennefeld, M., \& Voges, W. 1998, A\&AS, 129, 87 Brinkmann, W., Laurent-Muelheisen, S. A., Voges, W., et al. 2000, A\&A, 356, 445

Dickey, J. M., \& Lockman, F. J. 1990, ARA\&A, 28, 215

Fabbiano, G., Kim, G. W., \& Trinchieri, G. 1992, ApJS, 80, 531

Fischer, J. U., Hasinger, G., Schwope, A. D., et al. 1998, AN, 319, 347

Grupe, D., Beuermann, K., Mannheim, K., \& Thomas, H. C. 1999, A\&A, 350, 805

Grupe, D., Wills, B. J., Leighly, K .M., \& Meusinger, H. 2004, AJ, 127, 156

Hao, L., Strauss, M. A., Tremonti, C. A., et al. 2005, AJ, 129, 1783

Hasinger, G., Truemper, J., \& Schmidt, M. 1991, A\&A, 246, L2

Ho, L. C., Filippenko, A. V., \& Sargent, W. 1997, ApJ, 487, 568

Kewley, L. J., Dopita, M. A., Sutherland, R. S., et al. 2001, ApJ, 556, 121

Moran, E. C., Halpern, J. P., \& Helfand, D. J. 1996, ApJS, 106, 341

Motch, C., Guillout, P., Haberl, F., et al. 1998, A\&AS, 132, 34

Osterbrock, D. E. 1987, Lecture Notes in Physics, Active Galactivc Nuclei (Heidelberg: Springer)

Osterbrock, D. E., \& Pogge, R. W. 1985, ApJ, 297, 166

Paturel, G., Fouque, P., Bottinelli, L., \& Gouguenheim, L. 1989, A\&AS, 80, 299

Paturel, G., Petit, C., Prugniel, P., et al. 2003, A\&A, 412, 45

Pietsch, W., Bischoff, K., Boller, T., et al. 1998, A\&A, 333, 48 (Paper I)

Simcoe, R., McLeod, K. K., Schachter, J., \& Elvis, M. 1997, ApJ, 489, 615

Turnshek, D. A., Bohlin, R. J., Williamson, R. I., et al. 1990, AJ, 99, 1243

Vaughan, S., Edelson, R., Warwick, R., et al. 2001, MNRAS, 327, 673

Veron-Cetty, M. P., \& Veron, P. 2006, VizieR Online Data Catalogue 7248.0

Voges, W. 1993, AdSpR, 13, 391

Voges, W., Aschenbach, B., Boller, Th., et al. 1999, A\&A, 349, 389

Williams, R. J., Pogge, R. W., \& Mathur, S. 2002, AJ, 124, 3042

Xu, D. W., Wei, J. Y., \& Hu, J. Y. 2001, Chinese J. Astron. Astrophys., 1, 46

Zhou, H., Wang, T., Yuan, W., et al. 2007, ApJ, 658, L13

Zimmermann, H.-U., Boller, T., Doebereiner, S., \& Pietsch, W. 2001, A\&A, 378, 30 